Mariola Teresa Kozubek*

ORCID: 0000-0002-1377-3657

Katowice, Polonia

\title{
L'amore come dinamica fondamentale dell'educazione in famiglia secondo Chiara Lubich. Alcuni aspetti
}

\section{Love as the Fundamental Dynamics of Family Upbringing according to Chiara Lubich - Selected Aspects}

\begin{abstract}
Summary: Civilisational progress and globalisation processes at the turn of the $20^{\text {th }}$ and $21^{\text {st }}$ centuries have changed the shape, function and role of family in social life, posing a threat to its stability. Consequently, many family support institutions and organisations are created that offer social services focused on prevention, family therapy and support, particularly in terms of family upbringing. In search of a model of family upbringing that teaches how to build long-term interpersonal relationships and overcome difficulties by referring to values helpful in dealing with family crises, the article focuses on the pedagogical potential of contemporary
\end{abstract}

* Mariola Teresa Kozubek, PhD in Pedagogia. Docente di scienze dell'educazione e scienze della famiglia all'Università della Slesia a Katowice (Polonia); membro-esperto dell'Agenzia della Santa Sede per la Valutazione e la Promozione della Qualità delle Università e Facoltà Ecclesiastiche (AVEPRO); membro del Gruppo di Pedagogia Cristiana presso il Comitato delle Scienze Pedagogiche dell'Accademia Polacca delle Scienze. Indirizzo: Wydział Teologiczny US, ul. Jordana 18, 40-043 Katowice, Polonia. Mail: mariola.kozubek@us.edu.pl 
movements and communities of the Catholic Church. Substantial studies in this regard are provided by the New Families Movement, an initiative that operates under the international Focolare Movement founded by the Italian teacher Chiara Lubich (1920-2008). Based on the hermeneutics of Lubich's texts, the article presents her main thoughts on family upbringing. Following the adopted research procedure, the text discusses the significance of personal love from the philosophical, psychological and theological perspective as well as through life and educational experience of Chiara Lubich and her anthropological and theological reflections on education. In this context, several key aspects of family upbringing are discussed in the context of personal love: parental authority and responsibilities, specificity of integral education, characteristics of pro-social education and development of skills necessary to overcome difficulties. They appear to be a fundamental part in Lubich's 'style' of family upbringing. The resulting analysis belongs to the field of family pedagogy and pedagogy of religion, and is mostly based on Italian literature sources. Chiara Lubich's pedagogical thought is not well-known in Polish pedagogical literature, which makes it all the more compelling to address this topic.

Keywords: love in the family; education; Chiara Lubich.

Sommario: Lo sviluppo dei processi di civilizzazione e globalizzazione a cavallo tra il XX e il XXI secolo sta influendo sul cambiamento della forma della famiglia, della sua funzione e del suo ruolo nella vita sociale e costituisce una minaccia soprattutto per la sua stabilità. Per questo motivo, la famiglia viene sostenuta da molte istituzioni e organizzazioni di sostegno che operano nel campo dell'assistenza sociale per la prevenzione, la terapia e l'accompagnamento, in particolare nell'adempimento della sua funzione educativa. Nella ricerca di un modello di educazione nella famiglia che insegni a costruire relazioni interpersonali permanenti, a superare le difficoltà sulla base dei valori che aiutano ad affrontare le crisi della famiglia, l'attenzione è rivolta al potenziale pedagogico dei Movimenti e delle Comunità contemporanee della Chiesa cattolica. Un ricco materiale in questo senso lo fornisce il Movimento Famiglie Nuove - una delle branche del Movimento dei Focolari fondato da un'insegnante italiana: Chiara Lubich (1920-2008). Questo articolo presenta - sulla base dell'ermeneutica dei testi di Lubich - $\mathrm{i}$ suoi principali pensieri sull'educazione in famiglia. Nel procedere della ricerca, viene presentato il significato dell'amore secondo la prospettiva personalista dapprima nella prospettiva filosofica, psicologica e teologica, quindi nella vita e nell'esperienza educativa di Chiara Lubich e nel suo pensiero antropologico-teologico sull'educazione. In questo contesto vengono presentati alcuni aspetti chiave dell'educazione in famiglia sempre nel contesto dell'amore visto in 
chiave personalista. In particolare: il ruolo e l'autorevolezza genitoriale; la specificità dell'educazione integrale; le caratteristiche dell'educazione prosociale e la formazione delle abilità atte a superare le difficoltà. Essi, nello stile educativo secondo la visione di Lubich, sembrano fondamentali in famiglia. L'analisi condotta si colloca nel campo della pedagogia familiare e della pedagogia della religione e poggia principalmente sulla letteratura in lingua italiana. Nella letteratura pedagogica polacca il pensiero pedagogico di Chiara Lubich non è conosciuto, pertanto è ragionevole affrontare questo argomento.

Parole chiavi: amore in famiglia; educazione; Chiara Lubich.

La famiglia in quanto comunità fondamentale di vita ed educazione è oggetto di varie ricerche e dibattiti. Lo sviluppo della civiltà e i processi di globalizzazione avvenuti a cavallo tra il XX e XXI secolo hanno influito sul cambiamento del volto della famiglia, sulle sue funzioni e sul suo ruolo in ambito sociale. Si è arrivati, così, ad una certa universalizzazione a livello dei comportamenti che ha provocato con ciò una certa relativizzazione delle norme e di conseguenza la preferenza verso stili di vita 'di progresso'. Anche se, nella società polacca, $i$ risultati delle ricerche mostrano che in capo alla gerarchia dei valori dichiarati c'è sempre la famiglia $(97 \%, 1998,2012)^{1}$, stiamo assistendo ad una sua diffusa disgregazione. Dai dati della GUS (Ufficio Centrale di Statistica) risulta che - dopo un forte aumento dei divorzi fino al 2006 - negli ultimi anni questo fenomeno è effettivamente diminuito rimanendo stabile. Ma nonostante ciò, dal punto di vista pedagogico, non si è ridotta la preoccupazione riguardo lo sviluppo dei bambini nelle famiglie disintegrate, incomplete o ricostruite. Il motivo fondamentale per stabilire un divorzio è l'incompatibilità di carattere dei coniugi (nel 2017 questa è stata l'unica o una tra le varie ragioni per la separazione formale $)^{2}$. Di fronte a tutto ciò la famiglia ha, indubbiamente, bisogno di sostegno. Questo sostegno è assicurato da numerose istituzioni e organizzazioni di supporto che operano nel campo dell'assistenza sociale per la prevenzione, la terapia e l'ac-

${ }^{1}$ Cf. Wojciech Świątkiewicz, "Między rodziną a życiem publicznym - ciągłość i zmiana orientacji na wartości w polskim społeczeństwie", in: Postawy społeczno-religijne Polaków 1991-2012, ed. Lucjan Adamczuk, Elżbieta Firlit, Witold Zdaniewicz (Warszawa: Instytut Statystyki Kościoła Katolickiego SAC, 2013), 192-194.

2 Departament Badań Demograficznych, Rocznik Demograficzny (Warszawa: GUS, 2018), 235, 243. 
compagnamento. Vale la pena di notare che da diversi anni alcune università polacche preparano professionalmente degli assistenti familiari. Essi si occupano direttamente del lavoro con genitori e figli e indirettamente delle azioni attuate a beneficio dei bambini e della famiglia ${ }^{3}$. La famiglia viene sostenuta anche da varie comunità di tipo religioso, inclusa la Chiesa cattolica ${ }^{4}$ che la considera la sua prima e più importante via ${ }^{5}$.

Nel supporto completo alla famiglia un ambito particolare dovrebbe essere quello che riguarda le attività proprie della funzione educativa ${ }^{6}$. Nella ricerca di un modello di educazione in famiglia che insegni a costruire relazioni interpersonali a lungo termine e a superare le difficoltà sulla base dei valori, vale la pena di prestare attenzione al potenziale educativo dei moderni Movimenti e Comunità ecclesiali. I papi Giovanni Paolo $\mathrm{II}^{7}$, Benedetto XVI e Francesco ${ }^{9}$ - per i quali la famiglia è la prima e la più importante comunità educativa ${ }^{10}$ e il modello per tutti gli altri gruppi sociali - hanno incoraggiato e incoraggiano le comunità cristiane a sostenere la missione educativa

${ }^{3}$ Cf. Aleksandra Kłos-Skrzypczak, "Nauki o rodzinie w perspektywie amerykańskiego szkolnictwa wyższego", Studia Teologiczne i Humanistyczne 1/2 (2011): 247-258; Irena Krasiejko, Praca socjalna w praktyce asystenta rodziny. Przyktad podejścia skoncentrowanego na rozwiązaniach (Katowice: Wyd. Naukowe Śląsk, 2011). Il compito dell'assistente familiare stabilisce: art. 15 ustawy z dnia 9 czerwca 2011 o wspieraniu rodziny i systemie pieczy zastępczej (Dz.U. z 2018 r. poz. 998). Cf.: Rozporzadzenie Ministra Pracy i Polityki Spolecznej z dnia 9 grudnia 2011 r. w sprawie szkoleń na asystenta rodziny (Dz.U. z 2011 r. nr 272, poz. 1608).

${ }^{4}$ Katolickie poradnictwo rodzinne w Polsce w r. 2016 (Warszawa: Instytut Statystyki Kościoła Katolickiego, 2017), 2-15; Małgorzata Słowik, "O potrzebie rozwoju poradnictwa rodzinnego opartego na wartościach chrześcijańskich - na przykładzie działalności Kościoła katolickiego w Polsce", Paedagogia Christiana 2/30 (2012): 193-213.

5 Janusz Tarnowski, "Wprowadzenie do Deklaracji o wychowaniu chrześcijańskim", in: Sobór Watykański II, Konstytucje, Dekrety, Deklaracje (Poznań: Pallotinum, 1968), 309.

${ }^{6}$ Cf. Michel Robert Bous, Imparare ad amare (Magnano: Edizioni Qiqajon, 2008), 21.

7 Giovanni Paolo II, Esortazione apostolica 'Familiaris consortio' (Città del Vaticano: Libreria Editrice Vaticana, 1981), n. 72.

${ }^{8}$ Benedetto XVI, Messaggio ai partecipanti al II Congresso Mondiale dei Movimenti ecclesiali e delle Nuove comunità. 22 Maggio 2006 (Città del Vaticano: Libreria Editrice Vaticana, 2006); http://w2.vatican.va/content/benedict-xvi/it/messages/pont-messages/2006/ /documents/hf_ben-xvi_mes_20060522_ecclesial-movements.html.

9 "Qualsiasi altro collaboratore nel processo educativo deve agire in nome dei genitori, con il loro consenso e, in una certa misura, anche su loro incarico" [Francesco, Esortazione apostolica postsinodale Amoris Laetitia (Città del Vaticano: Libreria Editrice Vaticana, 2015), n. 84].

${ }^{10}$ Francesco, La Famiglia. Educazione. Udienza generale, 20.05.2015 (Città del Vaticano: Libreria Editrice Vaticana, 2015), 41. 
della famiglia secondo il principio di sussidiarietà. In Polonia tale compito è svolto dalla pastorale della famiglia e dai Movimenti e dalle Associazioni, tra cui Chiesa Domestica (Domowy Kościół) del Movimento Luce-Vita, Famiglia di famiglie (Rodzina Rodzin), Movimento delle Famiglie Nazaretane (Ruch Rodzin Nazaretańskich), Alleanza delle Famiglie (Przymierze Rodzin), Associazione delle Famiglie Cattoliche e Famiglie Nuove. L'analisi dei loro principi di formazione e delle loro attività educative dimostra che, indipendentemente dal carattere specifico di un dato carisma o spiritualità, essi riconoscono che la dinamica più importante della famiglia è l'amore, per cui la loro azione è quella di educare nell'amore e all'amore ${ }^{11}$. Un ricco materiale al riguardo ce lo fornisce il Movimento Famiglie Nuove del Movimento dei Focolari fondato da un'insegnante italiana: Chiara Lubich $(1920-2008)^{12}$. Esso è presente in oltre 180 paesi con una azione che influisce a livello educativo e formativo, sulla famiglia, sui bambini e sui giovani di diverse culture, fedi e religioni, cosi come su persone di convinzioni non religiose. Un'analisi della biografia di Lubich mostra che si tratta di una donna dotata di un indubitabile genio e di un carisma specifico. Di ciò danno prova, anche, numerose opere educative tra cui quelle orientate alla famiglia avviate da lei nei vari continenti ${ }^{13}$. Giovanni Paolo II, rivolgendosi al Movimento dei Focolari nel 1984, ha sottolineato che il suo ethos è caratterizzato dal radicalismo dell'amore: "È bene che abbiate trovato tale strada, questa vocazione all'amore [...]. Questa è la vostra fede, la scintilla ispiratrice di tutto quello che si fa con il nome Focolari, di tutto quello che voi siete [...]. Ci sono stati nella storia della Chiesa tanti radicalismi dell'amore [...]. C'è anche il vostro radicalismo dell'amore, di Chiara, dei Focolarini. [...] L'amore è più forte" 14 .

${ }^{11}$ Cf. Tomasz Ożóg, "Wychowanie chrześcijańskie w kościelenych ruchach religijnych", in: Wychowanie chrześcijańskie. Między tradycją a współczesnością, ed. Alina Rynio (Lublin: Wyd. KUL, 2007), 953-963; Adam Wodarczyk, Prorok żywego Kościoła (Katowice: Emmanuel, 2008); Zbigniew Nosowski, Parami do nieba. Matzeńska droga do świętości (Warszawa: Więź, 2004); Mariola T. Kozubek, "Pedagogie współczesnych ruchów odpowiedzią na Familiaris consortio", Studia Teologiczne i Humanistyczne 1/2 (2011): 195-209.

${ }^{12}$ Armando Torno, PortarTi il mondo fra le braccia - Vita di Chiara Lubich (Roma: Città Nuova, 2011).

${ }^{13}$ Negli anni 2007-2009 ho svolto la ricerca in Europa, Africa e America del Sud. (Progetto di ricerca - Grant NN106 431033 del Ministero delle Ricerche e dell’Università in Polonia).

${ }^{14}$ Giovanni Paolo II, Discorso ai Membri del Movimento dei Focolari. Centro 
Anche papa Francesco riconosce nei Focolari: "Una famiglia in cui tutti si riconoscono figli e figlie dell'unico Padre, impegnati a vivere tra loro e verso tutti il comandamento dell'amore reciproco"15. E perciò incoraggia loro a rimanere fedeli a questo ideale che porta: "Alla ricerca dell'unione con Dio e nell'amore vicendevole coi fratelli e le sorelle, attingendo alle ricchezze della Parola di Dio e della Tradizione della Chiesa, a questo anelito di comunione e di unità che lo Spirito Santo ha suscitato per il nostro tempo. E fate dono a tutti di questo tesoro!"'16.

Ed è proprio scopo di questo articolo presentare il pensiero di Chiara Lubich sull'educazione in famiglia nella dinamica dell'amore. Poniamo, quindi, una domanda ermeneutica sull'importanza dell'amore nell'educazione familiare e cerchiamo le risposte nei "testi" di Lubich, partendo dal presupposto che - come sottolinea Barbara Adamczyk, d'accordo con il pensiero di Jan Galarowicz sull'ermeneutica contemporanea - il termine "testo" può significare non solo una parola, un simbolo, un mito, ma anche "la fede $[\ldots]$, il comportamento umano, gli eventi, la realtà sociale" 17 . In tale approccio, l'ermeneutica stessa viene intesa come un metodo che descrive e interpreta il mondo umano letto come un "testo"18. Nell' approccio adottato diamo, quindi, lo sguardo dapprima al significato dell'amore in quanto base della formazione, in un secondo tempo ci rivolgiamo all'esperienza di vita, all'azione educativa e alla riflessione antropologica e teologica di Chiara $\mathrm{Lu}-$ bich circa l'educazione e, infine, presentiamo - come da lei proposto - uno 'stile' di educazione in famiglia. L'analisi presentata, situata nel campo della pedagogia della famiglia e della pedagogia della religione, mette in evidenza le specificità della proposta di Lubich nella percezione dell'amore quale fondamento dell'educazione nella famiglia.

internazionale «Mariapoli» - Rocca di Papa, Domenica, 19 agosto 1984 (Città del Vaticano: Libreria Editrice Vaticana, 1984).

15 Francesco, Discorso ai Patecipanti all'Asemblea Generale del Movimento dei Focolari. Sala Clementina, Venerdi, 26 settembre 2014 (Città del Vaticano: Libreria Editrice Vaticana, 2014).

${ }^{16}$ Ibidem.

17 Por. Jan Galarowicz, Na ścieżkach prawdy. Wprowadzenie do filozofii (Kraków: PAT, 1992), 43 [Cit. di: Barbara Adamczyk, Model pedagogii Jezusa w przekazie biblijnym (Kraków: WAM, 2008), 21].

${ }_{18}$ Teresa Bauman, Tadeusz Pilch, Zasady badań pedagogicznych (Warszawa: ZAK, 2018), 272. 


\section{L'amore come base dell'educazione. Breve descrizione del problema}

L'amore è colloquialmente un concetto ambiguo in genere ridotto al sentimento, ma in quanto categoria di grande profondità di significato è oggetto di analisi interdisciplinari. Dal punto di vista filosofico il concetto amore è collegato al bene, mentre da quello teologico alla realtà di Dio stesso ${ }^{19}$. L'amore nell'ottica dell' 'amore-dono' con la sua dimensione etica, ha delle implicazioni pedagogiche ed è immanentemente associato al processo educativo che scaturisce da una specifica antropologia. Per comprendere le dinamiche dell'amore in ambito pedagogico, occorre dargli un breve sguardo dal punto di vista filosofico, psicologico e teologico.

I concetti filosofici e le espressioni sull'amore risalgono già all'antichità. Basterebbe ricordare Platone e Aristotele, e più avanti, per esempio, san Tommaso d'Aquino, Descartes, Georg F. Hegel, Max Scheler, Gabriel Marcel, Dietrich von Hildebrandt e, tra i filosofi polacchi, Mieczysław A. Krąpiec e Władysław Stróżewski. Secondo Waldemar Furmanek, questi pensatori possono fornire molti spunti al tempo presente perché essi "vedono nell'amore stesso i valori pienamente costruttivi alla base del concetto di uomo in quanto essere amante" ${ }^{\prime 20}$. Analizzando l'amore come una caratteristica della vita personale, alla luce della filosofia realistica dell'uomo di Mieczysław A. Krapiec ${ }^{21}$, si sottolinea che l'amore può essere realizzato se "si pone l'intera vita dell'uomo nella prospettiva 'dell'essere-vivere per l'altra persona', e in questo senso l'amore determina lo stato relazionale dell'uomo", e penetrando profondamente nella struttura del suo essere personale, fa sì che "l'uomo si dona con tutto il suo bagaglio personale a un'altra persona e allo stesso tempo contribuisce alla co-creazione del bene in lei"22. "L'essere per gli altri" inteso in questo modo significa che "l'uomo è 'felice in se stesso'

19 Antoni Podsiad, Stownik terminów i pojęć filozoficznych (Warszawa: Pax, 2001), 514.

${ }^{20}$ Waldemar Furmanek, Miłość - zagubiona wartość wspótczesnej pedagogiki (Rzeszów: FOSZE, 2011), 79.

${ }^{21}$ Cf. Wojciech Chudy, "Krąpiec Mieczysław Albert”, in: Powszechna encyklopedia filozofii, vol. 6 (Lublin: TN KUL, 2005), 43-48.

${ }^{22}$ Barbara Kiereś, "Rodzina a problem aktualizacji przyrodzonej religijności człowieka. Na kanwie A.M. Krąpca realistycznej filozofii człowieka", in: Edukacyjny potencjat religii, ed. Jerzy Bagrowicz, Jarosław Horowski (Toruń: Wydawnictwo Naukowe UMK, 2012), 244-245. 
tanto quanto 'è per l'altra persona'"'23. Un significato simile dell'amore lo troviamo nell'ottica del personalismo cristiano. Emmanuel Mounier afferma che "una persona esiste solo rivolgendosi a un'altra persona, solo attraverso un'altra persona può conoscere se stesso, solo in un'altra persona può trovarsi [...]. Io esisto solo nella misura in cui io esisto per qualcun'altro, e, al limite, significa amare"24. Tale idea viene messa in evidenza da Giovanni Paolo II nella Lettera alle famiglie. Gratissimam sane: "L' «ethos» del personalismo è altruistico: muove la persona a farsi dono per gli altri e a trovare gioia nel donarsi" ${ }^{25}$. Gli approcci filosofici presentati danno una visione generale della dimensione pedagogica dell'amore: al saper-essere-un-dono-per-l'altro è indispensabile all'educazione.

L'analisi della categoria amore dal punto di vista della psicologia non è semplice, perché l'amore non è uno stato uniforme o permanente e nemmeno misurabile. La psicologia sociale riconosce l'amore come una relazione tra persone che vogliono fare del bene ad altre persone. Nelle ricerche psicologiche, l'amore viene analizzato anche nella prospettiva dei bisogni, dei sentimenti e degli atteggiamenti. Per Erich Fromm, che rappresenta la corrente della psicologia umanistica (la più vicina all'approccio personalistico sull'educazione all'amore), l'amore è un "modo di essere", e perciò come "l'atto d'amare" rivolto alla persona è "un'attività produttiva che implica l'occuparsi dell'altro, conoscere, rispondere, accettare, godere", diventando in questo modo, "un processo di autorinnovamento, di autoincremento" 26. Secondo Fromm amare significa avere un atteggiamento, una certa proprietà del carattere che determina la relazione di un uomo con il mondo in generale e non con un solo oggetto d'amore. Egli afferma anche che molte persone vogliono essere amate, non prestando però alcuna attenzione al fatto di dover e saper amare anche loro gli altri. Se è così, allora l'amore deve essere imparato e insegnato, perché se una persona ama unicamente un'altra persona, essendo indifferente nei confronti degli altri, allora "il suo non è amore, ma un attaccamento simbiotico o un egotismo portato all'eccesso" 27 . Si può presumere, quindi, che l'amore in tale approccio fa sì che l'educazione diventi

${ }^{23}$ Mieczysław A. Krapiec, Ja-człowiek (Lublin: KUL, 1998): 320-330 [Cit. di: Kiereś, Rodzina, 245].

${ }^{24}$ Emanuel Mounier, Wprowadzenie do egzystencjalizmów (Kraków: ZNAK, 1964), 37.

${ }^{25}$ Giovanni Paolo II, Lettera alle famiglie. Gratissimam sane (Città del Vaticano: Libreria Editrice Vaticana, 1994), n. 14.

${ }^{26}$ Erich Fromm, Avere o essere (Milano: Mondadori, 1977), 29.

27 Erich Fromm, L'arte di amare (Milano: Mondadori, 1986), 29, 50, 77. 
un atto d'amore ed essa - come scrive Wanda Półtawska - dovrebbe essere intrapresa sin dai primi momenti della vita del bambino ${ }^{28}$. Secondo lei, l'educazione all'amore in quanto dono, è una base indispensabile per preparare le giovani generazioni al saper creare in un futuro la propria famiglia ${ }^{29}$.

Data la prospettiva di questo articolo è importante dare uno sguardo anche al concetto cristiano dell'amore nell'educazione. L'amore nelle sue varie manifestazioni fino all'eroismo è il fondamento del cristianesimo e ne dà prova l'intero insegnamento di Gesù di Nazaret presentato nel Nuovo Testamento. Il comandamento dell'amore di Dio e del prossimo, lungo i secoli e fino ai nostri giorni, è invariabilmente il punto di riferimento per un umanesimo più profondo, e indica allo stesso tempo l'ideale e lo scopo dell'educazione in famiglia. Giovanni Paolo II sottolineando la necessità di considerare "l'uomo come un valore particolare e autonomo, come il soggetto portatore della trascendenza della persona" poneva come condizione la necessità di affermare l'uomo per se stesso, di rivendicare l'amore per l'uomo in ragione della dignità particolare che egli possiede ${ }^{30}$. Tale approccio gli proveniva dalla sua visione della norma personalistica ${ }^{31}$. Egli ha sottolineato anche che, nel definire l'educazione, è necessario considerare due verità fondamentali: "l'uomo è chiamato a vivere nella verità e nell'amore" e "ogni uomo si realizza attraverso il dono sincero di sé", e questo vale - spiega il Papa - sia per chi educa, sia per chi viene educato. In tale impostazione il processo di educazione in famiglia diventa una reciproca «elargizione» di umanità ${ }^{32}$.

${ }^{28}$ Wanda Półtawska, "Prawidłowy start", in: Miłość, małżeństwo, rodzina, ed. Franciszek Adamski (Kraków: Wyd. Apostolstwa Modlitwy, 1978), 44-45; Wanda Półtawska, Uczcie się kochać (Poznań: Edycja Świętego Pawła, 2015).

29 Wanda Półtawska, "Rola rodziców w wychowaniu seksualnym młodzieży", in: Wychowanie w rodzinie, ed. Franciszek Adamski (Kraków: Petrus 2010), 206; vedi: Jarosław Horowski, "Rola rodziny w wychowaniu seksualnym dzieci i młodzieży (w świetle dokumentów Stolicy Apostolskiej)", Paedagogia Christiana 1/9 (2002): 179-188.

${ }^{30}$ Giovanni Paolo II, Discorso all'Organizzazione delle Nazioni Unite per L'Educazione, la Scienza e la Cultura (UNESCO). Parigi, 2 giugno 1980 (Città del Vaticano: Libreria Editrice Vaticana, 1980), n. 10.

${ }^{31}$ La norma personalistica di Wojtyła indica il senso dell'educazione nella famiglia. Essa dice: "Ogni volta che nella tua condotta una persona è oggetto della tua azione, non dimenticare che non devi trattarla soltanto come un mezzo, come uno strumento, ma tieni conto del fatto che anch'essa ha, o perlomeno dovrebbe avere, il proprio fine" [Karol Wojtyła, "Amore e responsabilità", in: Karol Wojtyla, Metafisica della persona. Tutte le opere filosofiche e saggi integrativi (Milano: Bompiani, 2003), 478].

32 Giovanni Paolo II, Gratissimam, n. 16. 
Un ampio approccio alla comprensione dell'amore, sia in senso funzionalistico che in quello oggettivo, è stato compiuto da Benedetto XVI nell'enciclica Deus caritas est. Essa già nel titolo stesso include il significato ontologico dell' amore: "Dio è amore; chi sta nell'amore dimora in Dio e Dio dimora in lui" $(1 G v 4,16)^{33}$. L'amore cristiano ha dunque dimensione personale: è Dio-Amore. Benedetto XVI riferendosi all 'Inno alla carità della Prima Lettera ai Corinzi (1 Cor 12, 31-13, 8) spiega che la carità deve esprimersi nell'attività concreta ${ }^{34}$, e ciò va, però, insegnato, perché anche i più grandi valori del passato non vengono, semplicemente, ereditati. Benedetto XVI ci ricorda che l'amore dei genitori è "la prima e fondamentale esperienza dell'amore che i bambini fanno", e perciò l'educazione autentica sta nel sostenere i figli: (1) nella "ricerca della verità" di cui fa parte anche la sofferenza. Essa infatti aiuterà loro ad essere capaci di autentico amore e a superare gli egoismi; (2) nel tendere a "trovare un giusto equilibrio tra la libertà e la disciplina" e ciò coincide con quell'autorevolezza che "rende credibile l'esercizio dell' autorità" ed è sempre pronta "a correggere idee e scelte sbagliate"; (3) nel formare alla responsabilità sia l'educatore che l'educando avendo, però, sempre di vista anche la dimensione della famiglia umana ${ }^{35}$.

Continuando sulla linea dei suoi predecessori, anche papa Francesco constata che: "Educare è un atto d'amore, è dare vita. E l'amore è esigente, chiede di impegnare le migliori risorse, di risvegliare la passione e mettersi in cammino con pazienza insieme ai giovani" ${ }^{\prime 3}$. Secondo lui, i figli, per la testimonianza dei genitori, imparano quell' amore che si esprime nella fedeltà, nell'onestà e nel sacrifico ${ }^{37}$. Papa Francesco riconosce nel Movimento dei Focolari un certo modello di educazione in diversi ambiti della vita umana:

Da qui possono attingere nuovo impulso, arricchendosi con la fantasia dell'amore e aprendosi alle sollecitazioni dello Spirito e della storia, i percorsi di

${ }^{33}$ Benedetto XVI, Encilica Deus caritas est (Città del Vaticano: Libreria Editrice Vaticana, 2005), n. 1.

${ }^{34}$ Ibidem, n. 34; cf. Luigi Guglielmoni, Fausto Negri, L'amore familiare secondo san Paolo (Leumann: ELLEDICI, 2009).

${ }^{35}$ Benedetto XVI, Lettera alla Diocesi e alla Città di Roma sul compito urgente dell'educazione. 21 gennaio 2008 (Città del Vaticano: Libreria Editrice Vaticana, 2008), 4.

${ }^{36}$ Francesco, Ai partecipanti alla plenaria della Congregazione per L'Educazione Cattolica. Sala Clementina, 13 febbraio 2014 (Città del Vaticano: Libreria Editrice Vaticana, 2014), 2.

${ }^{37}$ Francesco, Festa delle Famiglie. Discorso del santo Padre Francesco. Croke Park Stadium (Dublino) Sabato, 25 agosto 2018 (Città del Vaticano: Libreria Editrice Vaticana, 2018). 
formazione che sono fioriti a Loppiano dal carisma dell'unità: la formazione spirituale alle diverse vocazioni; la formazione al lavoro, all'agire economico e politico; la formazione al dialogo, nelle sue diverse espressioni ecumeniche e interreligiose e con persone di diverse convinzioni; la formazione ecclesiale e culturale ${ }^{38}$.

La pedagogia moderna, specie nella sua corrente personalistica, tratta sempre di più il carattere multidimensionale dell'amore. Esso si esprime, tra l'altro come: categoria della teoria dell'educazione, valore educativo, base dello sviluppo personale che permette di trovare il vero significato della vita, e anche come efficienza morale nell'educazione ${ }^{39}$. Particolarmente degni di nota sono gli studi di Waldemar Furmanek perché egli presenta un’approfondita analisi interdisciplinare del significato dell'amore in quanto categoria pedagogica $^{40}$.

\section{L'amore nell'esperienza di Chiara Lubich}

Un'analisi approfondita dell'atto dell'uomo in quanto soggetto che agisce - dice Karol Wojtyła ${ }^{41}$-, ci permette di capire più profondamente chi è l'uomo. Riferendosi a questo concetto antropologico, Marian Nowak osserva che anche in un' analisi pedagogica si può partire dal fatto che "l'uomo agisce" e quindi "accettiamo, come base, la sua esperienza e la compren-

38 Francesco, Incontro con la comunità del Movimento dei Focolari. Sagrato del Santuario Maria Theotokos a Loppiano (Firenze). Giovedi, 10 maggio 2018 (Città del Vaticano: Libreria Editrice Vaticana, 2018).

${ }^{39}$ Cf.: Jarosław Żyliński, Miłość i wychowanie (Poznań: W Drodze, 2016); Miłość jako sprawność moralna w wychowaniu, ed. Iwona Jazukiewicz, Ewa Rojewska (Szczecin: Uniwersytet Szczeciński, 2013); Oblicza miłości w pedagogice, ed. Marcin Białas, Dorota Ruszkiewicz (Łódź: Wyd. WSH-E, 2007); Luigi Secco, La pedagogia dell'amore (Roma: Città Nuova 2006); Michele De Beni, Comunicare per amare (Roma: Città Nuova, 2005); Dorota Kornas-Biela, Rodzina: źródto życia i szkoła miłości (Lublin: TN KUL, 2000); Gary D. Chapman, I cinque linguaggi dell'amore, trad. Marina e Franco Valenti (Torino: ELLEDICI, 2012).

${ }^{40}$ Cf. Furmanek, Miłość; Waldemar Furmanek, Rozwój rozumienia miłości (Rzeszów: Wyd. Oświatowe FOSZE, 2011).

${ }^{41}$ Karol Wojtyła, "Teoria-prassi: un tema umano e cristiano", Rassegna di Pedagogia. Pädagogische Umschau 1-4 (2004): 17 [cit. di: Marian Nowak, "Wymiary teorii i praktyki w pedagogice w ujęciu Karola Wojtyły”, Wychowanie na co dzień 4-5(2005): 11]. 
sione di essa" e "in conseguenza di una tale analisi [...] la fonte basilare della conoscenza dell'uomo è il contatto cognitivo diretto dell'uomo con se stesso, che si svolge in un certo modo con il mondo coesistente" ${ }^{\prime 2}$. È proprio per comprendere profondamente il pensiero della Lubich sul significato dell'amore in famiglia, fondato su categorie antropologico-teologiche e in una particolare pedagogia, che occorre conoscere la sua esperienza personale e familiare contestualizzata in una concreta situazione storico-politica dell'Italia dell'inizio del XX secolo. Questo, perché l'uomo "vive sempre secondo una cultura che gli è propria, e che, a sua volta, crea fra gli uomini un legame che pure è loro proprio, determinando il carattere inter-umano e sociale dell'esistenza umana. [...] La cultura è ciò per cui l'uomo in quanto uomo diventa più uomo, «è» di più, accede di più all' «essere»" ${ }^{43}$. Nino Carella, autore di una ricerca biografica sul periodo giovanile di Chiara Lubich, sottolinea che sulla forma della vita dell'uomo e della cultura da lui creata, oltre ai fatti materiali influisce anche la dimensione teologale, e lo afferma con le parole della stessa Lubich: "piace al Signore deporre i suoi doni nel cuore di uomini e donne semplici, poveri, fragili, [...] perché meglio risplenda la sua potenza" 44 .

Silvia Maria Elvira Lubich - ovvero Chiara ${ }^{45}$ - è nata a Trento il 22 gennaio 1920 in una famiglia molto unita. Questo fatto - come dice lei stessa ha dato un'impronta significativa alla sua formazione e ha messo basi solide per il suo futuro: la fede forte e i principi morali trasmessi dalla madre, il senso della coerenza nella vita ricevuto dal padre e inoltre, per quanto riguarda la formazione religiosa, la conoscenza del catechismo ricevuta dalle suore della Congregazione di Maria Bambina a Trento. Incantata dalla sapienza, Lubich dedicava anche tempo all'approfondimento della propria fede e alla formazione delle aspiranti dell'Azione Cattolica. Nella corrispondenza tenuta con loro si possono già intravedere - come osserva Lucia Abignente - dei segni del carisma dell'unità. Già allora Lubich invitava all'amore a Gesù

${ }^{42}$ Nowak, "Wymiary teorii", 11.

43 Giovanni Paolo II, Discorso all'Organizzazione, n. 6-7; cf. Nino Carella, Silvia prima di Chiara. La ricercha di una strada nuova (Roma: Città Nuova, 2014), 11.

${ }^{44}$ Chiara Lubich, "Chi beve l'acqua pensa alla sorgente", in: Da Trento al mondo, dal mondo a Trento. Chiara Lubich nella sua città, ed. Michele Zanzucchi (Roma: Città Nuova, 2002), 77-83 [cit. di Carella, Silvia, 11].

45 Il nome Chiara l'ha preso all'inizio degli anni '40 del XX secolo, affascinata dalla figura di Santa Chiara d'Assisi [Cf. Chiara Lubich, Franca Zambonini, L'avventura dell'unità (Milano: Paoline Cinisello Balsamo, 1991), 41; Giulia Eli Folonari, Lo spartito scritto in cielo. Cinquant'anni con Chiara Lubich (Roma: Città Nuova, 2012), 27-45]. 
nell'altro, all'amare tutti, all'amore reciproco e all'unità ${ }^{46}$. Nell'anno 1938 diventa insegnante, dapprima nella Scuola elementare in Varollo di Livo, quindi nell'orfanotrofio presso l'Opera Serafica a Cognola e poi a Trento. Nei ricordi dei suoi allievi ed educandi Lubich si presenta come una educatrice di alta cultura personale, sensibile ai loro bisogni, empatica nelle relazioni ${ }^{47}$. Curava tanto l'educazione religiosa dei suoi allievi: ogni settimana proponeva loro una frase del Vangelo e quindi tutti i giorni raccontava loro gli effetti dell'attuazione di quella frase nella propria vita, inclusi gli insuccessi. In questo modo diventava più vicina a loro e con tanto amore dava coraggio per non fermarsi davanti agli ostacoli e invitava a superare le difficoltà ${ }^{48}$.

I momenti chiave della vita di Lubich, che hanno influito decisamente sul suo pensiero sulla famiglia e sulla comprensione del senso dell'amore nell'educazione, riguardano due eventi caratterizzati da un'esperienza misti$\mathrm{ca}^{49}$ : nell'anno 1939, durante un convegno delle studentesse cattoliche a Loreto, intravede nel modo di vivere della famiglia di Nazareth ${ }^{50}$ una "nuova" via. Il secondo episodio risale agli inizi degli anni ' 40 , quando Lubich sperimenta profondamente che Dio è Amore e la ama immensamente:

In mezzo al furore della guerra frutto dell'odio, Dio si è manifestato a noi, sotto l'azione di una grazia particolare, per quello che Egli veramente è: Amore. [...] È stata come una folgorazione [...] Dio è Amore, Egli dunque ci ama. Allora tutto ciò che accade, gioie e dolori, tutto è previsto da Lui, tutto è voluto, o permesso, dal suo amore. E la nostra vita ha acquistato un significato nuovissimo ${ }^{51}$.

${ }^{46}$ Lucia Abignente, Memoria e presente. La spiritualità del Movimento dei Focolari in prospettiva storica (Roma: Città Nuova, 2010), 70.

47 Torno, Chiara, 14; cf. Michele De Beni, Essere educatori. Coraggio di una presenza (Roma: Città Nuova, 2013).

${ }^{48}$ Lubich, L'avventura, 40.

49 Esperienza mistica compresa come un processo o uno stato di carattere [tipo] affettivointellettuale, nel quale il mistico è convinto sul suo contatto diretto con Dio e della sua presenza, del suo agire e dell'unione con Lui dentro di sé [Cf. Jakub Filek, "Doświadczenie mistyczne", in: Encyklopedia Katolicka, vol. 4, ed. Ludomir Bieńkowski, Feliks Gryglewicz, Romuald Łukaszyk (Lublin: TN KUL, 1983), 154].

${ }^{50}$ Questa nuova famiglia è diventata il focolare (it. fuoco domestico), una comunità di laici che dedicano la propria vita a Dio restando nel mondo [Lubich, L'avventura, 44; Chiara Lubich, La dottrina spirituale. Nuova edizione aggiornata e ampliata, ed. Michele Vandeleene (Roma: Città Nuova, 2009), 94-97].

${ }^{51}$ Cf. Lubich, L'avventura, 46-47. 
Questi eventi portano delle conseguenze: nell'anno 1944, a causa di un forte bombardamento di Trento, la maggioranza dei suoi abitanti (tra cui la famiglia Lubich) abbandona la città, ma lei vi rimane per vivere per la sua "nuova famiglia", e cioè per la nascente comunità dei Focolari ${ }^{52}$. L'arcivescovo di Trento, Carlo De Ferrari vede, nell'atteggiamento di questa giovane cristiana e della comunità radunata attorno a lei che tendeva all'unità evangelica, l'opera di Dio (in latino: "digitus Dei est hic" - "qui c'è il dito di Dio"), e da la sua approvazione al Movimento nascente ${ }^{53}$, nonostante l'atteggiamento scettico delle autorità ecclesiastiche verso le novità portate dai laici.

Un'altra tappa significativa per lo sviluppo del pensiero di Lubich sulla famiglia è l'incontro con Igino Giordani (1894-1980), avvenuto nel 1948 a Roma. Giordani, uomo di alta cultura (autore di oltre 88 publicazioni), esperto dell'insegnamento sociale della Chiesa, apprezzato nel mondo politico, deputato al parlamento italiano, ma anche sposato, padre di quattro figli, intravede nella giovane Lubich un "genio" nel senso sia umano che spirituale ed ecclesiale. Così la descrive:

Essa metteva la santità a portata di tutti; toglieva via i cancelli che separano il mondo laicale dalla vita mistica. [...] Avvicinava Dio: lo faceva sentire Padre, fratello, amico, presente all'umanità. [...] Una cosa avvenne in me. [...] Era successo che l'idea di Dio aveva ceduto il posto all'amore di Dio, l'immagine ideale al Dio vivo ${ }^{54}$.

Giordani donerà al Movimento nascente tutta la richezza della propria spiritualità, esperienza e pensiero, venendo riconosciuto, in seguito, come suo confondatore ${ }^{55}$. Ed è stato per la collaborazione con lui che nell'anno 1959 Lubich fonda il Centro san Giovanni Bosco diventato poi una piattaforma di scambio di riflessioni ed esperienze sulla prassi educativa delle persone legate al Movimento dei Focolari e sulla ricerca di una pedagogia

${ }^{52}$ Ibidem, 55; Chiara Lubich, Lettere dei primi tempi (1943-1949) alle origini di una nuova spiritualità (Roma: Città Nuova, 2010).

${ }_{33}$ Cf. Lucia Abignente, Qui c'è il dito di Dio. Carlo de Ferrari e Chiara Lubich: il discernimento di un carisma (Roma: Città Nuova, 2017); Lubich, L'avventura, 55.

${ }^{54}$ Igino Giordani, Memorie di un cristiano ingenuo (Roma: Città Nuova, 1981), 149-150.

55 Ibidem, 147-165; Chiara Lubich, "Igino Giordani: il confondatore", Nuova Umanità 1 (1995): 5-10. Egli descrive l'influsso del carima dell'unità sulla vita familiare e sociale in: Igino Giordani, Famiglia comunità d'amore (Roma: Città Nuova, 1969); Igino Giordani, La famiglia. Ricordi, pensieri (Roma: Città Nuova, 2005); Chiara Lubich, Igino Giordani, «Erano i tempi di guerra...». Agli albori dell'ideale dell'unità (Roma: Città Nuova, 2007), 133-139. 
poggiata sull'amore. In Italia, nel mondo scolastico del dopoguerra mancava un chiaro riferimento all'insegnamento della Chiesa cattolica sull'educazione. Questo Centro ha rappresentato una risposta concreta a tale lacuna. Tommaso Sorgi, sociologo italiano impegnato fin dal primo momento in esso, era convinto che la parola del Vangelo "ogni volta che avete fatto queste cose a uno solo di questi miei fratelli più piccoli, l'avete fatto a me" $(M t 25,40)$ avrebbe potuto essere la chiave interpretativa ai problemi educativi per gli insegnanti, i genitori, ma anche per i leader impegnati nella formazione nelle organizzazioni e comunità di tipo religioso. Egli riteneva che se il loro agire educativo avesse poggiato sulla collaborazione con Dio, sarebbe diventato espressione del Suo amore verso gli educandi ${ }^{56}$. Al Centro san Giovanni Bosco si era convinti che l'unità cristiana dovesse informare anche la pedagogia $^{57}$. Giuseppe M. Zanghí, filosofo di questo Centro, constatava che è un assurdo staccare l'uomo da Dio e spiegava cos'è la relazione interpersonale secondo l'idea dell'unità nel Movimento dei Focolari:

Si cerca l'unità, nel mondo. Si cercano rapporti nuovi. C'è un solo rapporto che sia d'unità vera: quello della SS. Trinità [...]. Se questo rapporto si attua in tutta la sua portata, sul piano quindi di operare, chè su quello dell'essere c'è sempre stato [...], non può non esprimere da sè strutture sociali tali da tradurlo nel loro linguaggio, tali da far dire, parafrasando il salmista, "le città degli uomini narrano la gloria di Dio!" Questo un modo di fare. Questo, siamo convinti, il motivo su cui intonare l'educazione dei giovani. Con una pedagogia che sia essenzialmente modo di vivere, modellato su quel meraviglioso "come Io e Te", cui più o meno conspaevolmente, il mondo tutto va orientandosis ${ }^{58}$.

Nelle linee-guida di questo centro pedagogico leggiamo, tra 1'altro, della necessità di riconoscere Gesù in quanto modello dell'insegnante "presente" nella comunità scolastica per l'amore reciproco, della formazione degli educandi attraverso l'amore che essi irradieranno anche nella famiglia e della collaborazione tra scuola e famiglia ${ }^{59}$.

Il fatto che tante famiglie abbiano aderito al Movimento ha fatto sì che nel 1967 sia nato in seno ad esso una diramazione chiamata Movimento Fa-

56 Tommaso Sorgi, "Scuole del nostro tempo", Città Nuova 2 (1960): 11-12.

57 Pasquale Foresi, "Educatori a convegno", Città Nuova 1 (1960): 6-7.

58 Giuseppe M. Zanghì, "Pedagogia nuova", Città Nuova 2 (1960): 11.

59 Centro San Giovanni Bosco. Storia istituzionale (Rocca di Papa: Archivio Chiara Lubich). 
miglie Nuove ${ }^{60}$ così come l'anno prima era nato il Movimento Gen (Generazione Nuova) per bambini, adolescenti e giovani ${ }^{61}$. In risposta alla crescente necessità di una formazione sistematica, col tempo Lubich fonda "scuole" di formazione per famiglie, bambini e giovani ${ }^{62}$. Un areopago del tutto particolare, nel quale lei forma le famiglie di ogni estrazione culturale, presentando loro l'amore come fondamento della vita di famiglia e forza educativa, sono i periodici congressi internazionali di formazione per famiglie, che si svolgono a Roma. Così come i suoi tanti messaggi alle famiglie ${ }^{63}$, ai bambini e ai giovani ${ }^{64}$.

Il carisma dell'unità profondamente poggiato sull'insegnamento della Chiesa cattolica trova in essa piena approvazione. Chiara Lubich diventa consultrice del Pontificio Consiglio per i Laici (1985), partecipa anche ad alcuni Sinodi dei Vescovi: in occasione del XX del Concilio Vaticano II (1985), sulla vocazione e i compiti dei laici (1987), sulla Chiesa in Europa (1990 e 1999).

Il valore educativo del pensiero e dell'agire educativo di Lubich viene quindi percepito dagli ambienti accademici. Nell'anno 1996, l'Univer-

${ }^{60}$ Chiara Lubich, Dove la vita si accende. Dialoghi sulla famiglia. (Roma: Città Nuova, 1999); Tratti di storia del Movimento Famiglie Nuove, ed. Danilo e Anna Maria Zanzucchi (Roma: Città Nuova, 2005); Raffaella Pinassi Cardinali, I focolarini sposati, una "via nuova" nella Chiesa (Roma: Città Nuova, 2007); Colomba In Hye Kim, Gli sposi e la famiglia in Igino Giordani. Pensiero e testimonianza (Roma: Città Nuova, 2011).

${ }^{61}$ Cf. Chiara Lubich, Colloqui con i gen. Anni 1966/1969 (Roma: Città Nuova, 1998), 7-10; Movimento dei Focolari, Paolo VI al Movimento dei Focolari (Roma: Città Nuova, 1978), 32 .

${ }^{62}$ Esse sono centri di formazione situati nelle cosidette 'cittadelle' del Movimento: in Italia, Germania, Argentina, Kenia, Camerun, negli Stati Uniti. I membri del Movimento vi stazionano temporaneamente [vedi: Gianni Bianco, Una famiglia grande come il mondo (Roma: Città Nuova, 2008)]. Il nome 'scuola' si riferisce anche ai brevi corsi (durata massima di due settimane), o convegni tenuti nelle varie zone/aree geografiche del Movimento. In essi, oltre alla formazione alla spiritualità del Movimento, sono previste anche lezioni che riguardano l'insegnamento della Chiesa cattolica sulla famiglia e vari argomenti in prospettiva pedagogica, psicologica e sociologica.

${ }^{63}$ Vedi: discorsi di Chiara Lubich ai convegni mondiali delle famiglie: a Roma (1981): "La famiglia e l'amore"; a Roma (1993): "Come la famiglia, così la società"; a Lucerna (1999): "La famiglia è il futuro" [vedi: Lubich, La dottrina, 263-267; 267-269; 270-281].

${ }^{64} \mathrm{Il}$ dialogo educativo di $\mathrm{Ch}$. Lubich con i giovani, ragazzi e bambini è stato pubblicato da Città Nuova: Colloqui con i GEN. Anni 1966/69 (Roma, 1998); Colloqui con i GEN. Anni 1970/74 (Roma, 1999); Colloqui con i GEN. Anni 1975/2000 (Roma, 2001); Ai Gen 3. 1971-1975 (Roma, 1979); Ai Gen 3. 1975-'80 (Roma, 1994); Ai Gen 3. 1981-1995 (Roma, 2006). 
sità Cattolica di Lublino le ha consegnato il dottorato honoris causa per le scienze sociali ${ }^{65}$ e negli anni seguenti altre 15 università le hanno conferito il grado di dottore honoris causa, tra cui l'Università Cattolica d'America, a Washington DC, per la Pedagogia (2000) ${ }^{66}$. Il contributo di Lubich al dialogo, al rinnovamento della società e della famiglia viene notato anche da istituzioni internazionali che le conferiscono, tra l'altro: il Premio Templeton (Londra, 1977), il Premio per l'Educazione alla Pace (Unesco, 1996), il Premio dei Diritti della Persona (Strasburgo, 1991). L'ultima opera fondata da Chiara Lubich è stata l'Istituto Universitario Sophia, approvato il 7 dicembre 2007 dalla Congregazione per l'Educazione Cattolica e inaugurato il 1 dicembre 2008 ${ }^{67}$. Il 14 marzo 2008, dopo una lunga malattia, Chiara Lubich muore nella sua residenza di Rocca di Papa attorniata dalla famiglia focolarina di tutto il mondo. Il 27 gennaio 2015, il vescovo di Frascati Raffaello Matinelli apre solennemente il processo canonico di beatificazione della Serva di Dio Chiara Lubich ${ }^{68}$.

Il pensiero di Chiara Lubich sulla famiglia emerge dalla sua profonda esperienza di famiglia intesa in senso lato e oltre i legami di sangue. Lo esprime in un suo testo intitolato Un Testamento: siate una famiglia ${ }^{69} \mathrm{e}$ anche in una intervista concessa al filosofo polacco Tadeusz Styczeń:

In confronto a quelli che mi seguono, mi sento madre, sorella, con tutta la pienezza dei sentimenti umani, e nello stesso tempo un "nulla" che deve permettere di passare su di loro la luce e la volontà di Dio. E così mi sente questa mia famiglia in tale riferimento che è inseparabilmente umano e divino. C'è tra di noi unità non solo effettiva ma anche profondamente affettiva che acquista valore divino per l'amore a Gesù Abbandonato ${ }^{70}$.

${ }^{65}$ Centro Chiara Lubich, Istituto Universitario Sophia, Dottorati honoris causa conferiti a Chiara Lubich. Laudationes, Motivazioni, Lezioni magistrali, ed. Florence Gillet e Rosalia Parlapiano (Roma: Città Nuova, 2016), 15-51.

${ }^{66}$ Ibidem, 309-319, 320-329.

${ }^{67}$ Cf. Piero Coda, "L'idea di Università di Chiara", in: La fonte e le frontiere. Sophia compie 10 anni (Città Nuova, 2018), 125-165; vedi: Maria Voce, "Uno strumento efficace di formazione alla cultura dell'unità", in: La fonte e le frontiere. Sophia compie 10 anni (Città Nuova, 2018), 17-21.

${ }^{68}$ Causa di beatificazione, https://www.focolare.org/chi-siamo/causa-di-canonizzazione/ [20.01.2019].

${ }^{69}$ Lubich, La dottrina, 92-93.

70 Tadeusz Styczeń, "Kochaj, gdyż Bóg nas wszystkich ukochał. Z Chiarą Lubich założycielką Ruchu Focolari rozmawia Tadeusz Styczeń SDS”, ETHOS 29 (1995): 17-18. 


\section{L'amore nel pensiero antropologico-teologico di Chiara Lubich}

Hans Urs von Balthasar constata che "ogni filosofia umana [...] è nello stesso tempo essenzialmente religiosa e teologica, poiché pone la domanda dell'essere assoluto" "71. Nel contesto dell'argomento affrontato in questo articolo tale pensiero viene completato dalla constatazione di Wiesław Jankowski il quale sottolinea che "la stessa realtà della famiglia e la natura dell'educazione in famiglia ci aprono ad accogliere le ispirazioni teologiche in pedagogia"72. È per questo che, per presentare lo specifico della pedagogia di Chiara Lubich, occorre prima mostrare le basi del suo pensiero antropologico-teologico. Esse sono coerenti con l'insegnamento del Concilio Vaticano II per il quale il senso dell'antropologia cristiana sta nel concetto dell'uomo rivelato da Gesù di Nazaret ${ }^{73}$. Riferendosi all'autocoscienza dell'uomo, ella spiega che nella carne e nell'anima di ogni uomo vi è un qualche cosa che dice che l'uomo è per Dio, e cioè che è "in comunione con Dio" perché è stato creato "per conoscere Dio" e "per amare Dio" ciò che è prossimo all'uomo (le realtà varie) e ciò che è in esso (l'interiorità) è lo spazio della sua esistenza. Ed è proprio questo essere "che è comune a tutte le realtà - e per il quale esse non sono un nulla - che rivela, in una manifestazione naturale, quell'Essere che nessuna di esse è, ma che in tutte si annuncia"75. Quindi "il loro divenire, i loro limiti, lo stesso cessare di esistere è il linguaggio nel quale viene detto che l'essere di tutto quanto esiste ha la sua radice in un Essere che semplicemente e assolutamente è”. Lubich ritiene che si potrebbe dire la stessa cosa, analogamente, sulla vita interiore dell'uomo: la sua autocoscienza, quando viene illuminata dalla fede, diventa un "riconoscimento dell'essere che nella coscienza è luce e, insieme, è confessione dell'Essere Assoluto", perciò - secondo lei - "il dire dell'uomo

${ }^{71}$ Hans Urs von Balthasar, La mia opera ed epilogo (Milano: Jaca Book, 1994), 88.

${ }^{72}$ Wiesław Jankowski, "Znaczenie wychowania integralnego dla odnowy rodziny”, Studia nad Rodzina ATK 1 (1997): 78.

${ }^{73}$ Concilio Vaticano II, Costituzione pastorale sulla chiesa nel mondo contemporaneo Gaudium et spes (Città del Vaticano: Liberia Editrice Vaticana, 1967), n. 22, 24; Lubich, Dottorati, 85.

${ }^{74}$ Lubich, Colloqui con i gen. Anni 1975/2000, 85.

75 Lubich, Dottorati, 164. 
«io»" significa "aprirsi per poter dire, nella comunione con l'essere di ogni cosa, che l'Essere Assoluto è"'76.

Chiara Lubich individua la dimensione religiosa dell'uomo a partire dalla sua immagine biblica e cioè in quanto 'immagine di Dio'77. Il suo legame con Dio - secondo lei - non è un qualcosa di aggiunto alla sua umanità, in quanto l'uomo è stato creato in tal modo, che lo stesso essere uomo include in sè il riferimento a $\mathrm{Dio}^{78}$. Quindi, se all'essenziale dell'umanità della persona appartiene "il legame con Dio nella dimensione dell'essere creato [...] allora egli - per realizzarsi pienamente - deve sperimentare tale relazione con Dio". Ciò avviene nel compiere la sua volontà e ciò significa "vivere come Gesù" "79, che ha mostrato la misura dell'amore all'uomo.

Il pensiero antropologico-teologico di Lubich, sopra descritto in breve, sta alla base del suo concetto di educazione. Jerzy Bagrowicz constata che "il modo di definire il fenomeno dell'educazione include un suo concetto ben preciso che dipende dalla visione dell'uomo, del mondo, della moralità e del posto dell'uomo nel mondo" $"$. La risposta a tale presupposto la si trova in un breve testo di Lubich espresso in un linguaggio immediato ed esistenziale: "Chi mi sta vicino è stato creato in dono per me ed io sono stata creata in dono a chi mi sta vicino. Sulla terra tutto è in rapporto di amore con tutto: ogni cosa con ogni cosa. Occorre però esssere l'Amore per trovar il filo d'oro fra gli esseri" ${ }^{81}$.

Secondo Lubich l'uomo nella sua natura relazionale ${ }^{82}$ è capace di essere dono grazie a un amore specificamente personale. Da tale presupposto emerge un compito di natura pedagogica: per saper accogliere un dono, e saper esserlo per gli altri, è indispensabile formare la persona all'amore. E questo avviene nell'itinerario "che il soggetto educando compie con l'aiuto dell'educatore" ${ }^{" 33}$. Il senso ontologico di questo amore - e cioè lo scopo dell'edu-

76 Ibidem.

77 Chiara Lubich, Tylko jedno (Katowice: Księgarnia św. Jacka, 1984), 140, 196.

${ }^{78}$ Cf. Claus Westermann, "Genesis, 1Teilband Genesis 1-11“, in: Biblischer Kommentar Altes Testament, vol. I (Vluyn: Neukirchen, 1974), 217-218 [cit. da Lubich, Tylko, 139, 196].

${ }^{79}$ Lubich, Tylko, 139-140.

80 Jerzy Bagrowicz, Stawać się bardziej człowiekiem. U podstaw edukacji religijnej (Toruń: Wyd. Naukowe UMK, 2008), 190.

${ }^{81}$ Lubich, La dottrina, 142.

${ }^{82}$ Cf. Jesús Morán, “'Trasferirsi nel fratello’: una radicalizzazione antropologica”, in: Guardare tutti i fiori. Da una pagina del '49 di Chiara Lubich (Roma: Città Nuova, 2014), 44-51.

${ }^{83}$ Lubich, Dottorati, 324. 
cazione - è così descritto da lei: "Nell'amore quel che vale è amare. Così su questa terra. L'amore - parlo dell'amore soprannaturale che non esclude il naturale - è una cosa tanto semplice e tanto complessa. Esige la tua parte ed aspetta la parte dell'altro" $"$.

\section{L'amore nell'educazione in famiglia secondo Chiara Lubich. Alcuni aspetti}

L'amore costituisce un principio interno che plasma e vivifica la famiglia come comunità di persone ${ }^{85}$. Alla luce delle riflessioni sopra presentate rivolgiamo, ora, l'attenzione verso alcuni aspetti che riguardano l'educazione in famiglia secondo Chiara Lubich: l'autorevolezza genitoriale e il ruolo dei genitori; l'educazione integrale; l'educazione pro-sociale e, infine, l'educazione al saper superare le difficoltà.

\subsection{L'amore, l'autorevolezza e il ruolo dei genitori}

L'autorevolezza genitoriale viene definita nell'ambito dell'autorità dei genitori nelle relazioni con i propri figli, attraverso la quale essi influiscono sulle loro convinzioni, decisioni e atteggiamenti. Essa deve essere esercitata in un'atmosfera d'amore e nel rispetto dei figli perché solo in tale maniera la loro libertà non viene limitata ed essi hanno la possibilità di saper discernere e agire. In ciò, un significato del tutto particolare riveste la testimonianza di vita dei genitori ${ }^{86}$. Papa Francesco constata che "lo sviluppo affettivo ed etico di una persona richiede un'esperienza fondamentale: credere che i propri genitori sono degni di fiducia"87, e sottolinea che nell'educazione è importante correggere ed ammonire i figli ${ }^{88}$. Secondo Lubich, in famiglia il considerare l'autorevolezza è una cosa naturale dato che essa manifesta l'amore dei

${ }^{84}$ Chiara Lubich, "Meditazioni" (1959), in: Chiara Lubich, Scritti Spirituali/1 (Roma: Città Nuova, $\left.1991^{3}\right), 48$.

${ }^{85}$ Giovanni Paolo II, Familiaris, n. 21.

${ }^{86}$ Sabina Bartel, “Autorytet rodzicielski”, in: Słownik matżeństwa i rodziny, ed. Edward Ozorowski (Warszawa-Lomianki: ATK, 1999), 31-32.

87 Francesco, Amoris, n. 263.

88 Jorge Mario Bergoglio, Wymagania i pasja. O wychowaniu chrześcijańskim, trad. Lidia Wrona (Kraków: Espirit SC, 2013), 22. 
genitori per i figli. Ella invita i bambini e i giovani ad amare i loro genitori senza limiti e ne da giustificazione riferendosi alla loro propria dimensione spirituale:

Amate tutti: specie mamma che vi presenta la Volonta di Dio. Obbeditela, amatela, servitela in nome dell'obbedienza che le è dovuta, e in nome dell'Ideale che rafforza in noi l'amore per Iddio e quindi per i prossimi [...]. Poi amate tutti. Per voi non ci sono eccezioni nell'amore [...] L'amore non subisce sconfitta mai”"89.

Circa il significato educativo dell'autorevolezza dei genitori Lubich ne parla nel contesto dei loro compiti formulati da lei sulla base della pedagogia di Gesù di Nazaret: (1) dare l'esempio: introdurre per primi nella propria vita tutto ciò che poi viene chiesto ai figli; (2) intervenire in aiuto concretamente: sapersi sacrificare per i propri figli in modo da innestare in loro l'amore soprannaturale; (3) lasciare libertà e dare fiducia: non imporre le proprie opinioni, ma donarle con amore; (4) correggere se occorre: l'ammonimento deve essere espresso con pace e calma; (5) manifestare la misericordia: perché essa «tutto copre» (1 Cor 13,7); (6) insegnare: sempre con amore ed esprimersi in modo chiaro; (7) condurre il dialogo: che deve essere aperto e creativo con una lettura precisa dei 'segni dei tempi'; (8) dire coraggiosamente la verità: presentando il cristianesimo in modo trasparente, con tutte le sue esigenze; (9) svolgere il ruolo dell'educatore: perché i figli hanno bisogno di modelli e direttive ben chiari ${ }^{90}$.

Nell'opinione di Katarzyna Olbrycht, Chiara Lubich risulta essere un'esperta coscienziosa di bisogni, problemi e difficoltà educative. A suo avviso, ciò è dimostrato dalle raccomandazioni concrete, comprensibili a tutti e sempre valide che ella propone in relazione ai compiti educativi dei genitori per i quali considera Gesù il miglior educatore ${ }^{91}$.

${ }^{89}$ Chiara Lubich, "Una schiera di giovanetti che segue Dio. Roma, 1 settembre 1953", in: Raccolta dei testi di Chiara ai ragazzi o sui ragazzi. Stralci da conversazioni, lettere, messaggi dal 1953 ad oggi per la formazione degli animatori del Movimento Ragazzi per l'unita, ed. Centro Ragazzi per l'unità (Rocca di Papa, 1999), 5; vedi: Chiara Lubich, Una via nuova. La spiritualità dell'unità (Roma: Città Nuova, 2002).

${ }^{90}$ Lubich, La dottrina, 308-312.

${ }^{91}$ Katarzyna Olbrycht, "Co może odnaleźć pedagog w zbiorze tekstów «Charyzmat jedności» Chiary Lubich?”, Nowe Miasto 2-3 (2008): 168. 


\subsection{L'amore nell'educazione integrale in famiglia}

L'uomo percepito come una realtà inviolabile considera anche l'amore come un'esperienza integrale - afferma Władysław Stróżewski e spiega che ciò vale per l'intelletto, per gli atti di volontà, per la decisione, per la responsabilità, per i sentimenti ${ }^{92}$. Papa Francesco sottolinea che è necessario "generare nel figlio, con molto amore, processi di maturazione della sua libertà, di preparazione, di crescita integrale, di coltivazione dell'autentica autonomia"93. Nello svilluppo integrale dell'uomo il significato fondamentale lo ha la famiglia ${ }^{94}$.

L'analisi di alcuni contenuti del programma di formazione nel Movimento dei Focolari mostra che l'educazione integrale vi è intesa come un'educazione globale così come risulta dalle ipotesi antropologiche adottate da Lubich. Ella sottolinea che l'amore, possedendo varie espressioni, si manifesta in vari modi, distinti tra loro e spiega che mediante questo approccio, esso penetra tutte le dimensioni educative della famiglia. Questa particolare modalità di declinazione dell'amore è stata definita da lei come: "vita di tutti gli aspetti" 95 , che nel contesto della teoria dell'educazione, esprime le realtà di tutte le sue dimensioni. Così lo descrive Lubich:

- "L'amore porta alla comunione, è comunione". In relazione all'educazione prosociale, si dovrebbe insegnare: a saper condividere beni spirituali e materiali; ad avere un atteggiamento adeguato verso il lavoro e l'economia percepiti come un bene comune e tutto ciò finalizzato a promuovere una cultura del dare e della solidarietà sociale.

- "L'amore non è chiuso in sè, ma per sua natura si apre agli altri". Queste sono indicazioni nel campo dell'educazione civica e patriottica che plasmano l'identità e l'amore per il proprio paese, ma anche l'apertura e l'ospitalità con un impegno che tende alla fratellanza

${ }_{92}$ Władysław Stróżewski, “Oblicza miłości”, Tygodnik Powszechny 6 (2000): 1 [cit. di Furmanek, Miłość, 85].

93 Francesco, Amoris, n. 261.

${ }^{94}$ Danuta Opozda, "Integral Upbringing as the Fundamental Category of Practice and Theory of Pedagogy of Family in Christian Inspiration", Paedagogia Christiana 2/42 (2018): $151-164$.

${ }^{95}$ Lubich, La dottrina, 258-261. 
universale. Un'interpretazione di tale atteggiamento è il concetto di "uomo-mondo" "96.

- "L'amore eleva l'anima”. Tale dovrebbe essere lo scopo dell'educazione religiosa e morale che consiste nel modellare il proprio carattere attraverso quei valori che sono focalizzati sulla ricerca della perfezione nell'amore di Dio e del prossimo.

- "L'amore risana". È l'obiettivo dell'educazione fisica ed ecologica che dovrebbe insegnare la cura della vita dal momento del concepimento alla morte naturale, la cura della salute in senso lato e la protezione dell'ambiente naturale percepito come una casa comune.

- "L'amore si esprime nell'armonia della comunità radunata". Nel processo di educazione alla dimensione artistica è necessario modellare la sensibilità alla bellezza e all'estetica.

- "L'amore è la fonte della sapienza". Considerato cosi l'amore rappresenta la sorgente dell'educazione intellettuale che conduce alla sapienza e rende capace della ricerca della verità e l'acquisizione del sapere per metterlo al servizio degli altri.

- "L'amore compone in uno i molti, è unità" ${ }^{97}$. Questo è l'obiettivo principale dell'educazione alla comunicazione che implica anche imparare a usare tutti i mezzi per diffondere il bene e costruire l'unità.

Queste sette espressioni dell'amore che abbracciano tutti gli aspetti della vita umana, sono anche un punto di riferimento nel processo di autoeducazione. Nel concetto di educazione integrale di Chiara Lubich, la cui fonte è il carisma dell'unità, si può pertanto intravedere la saggezza pedagogica della fede cristiana ${ }^{98}$.

\subsection{L'amore nell'educazione prosociale in famiglia}

L'amore è considerato una componente importante del comportamento umano e un elemento essenziale della vita dell'uomo ed è anche oggetto

${ }^{96}$ Lubich, Colloqui con i gen. Anni 1970/1974, 73-82; Mariola T. Kozubek, "Wspólnota i jedność w wybranych obszarach życia społecznego - doświadczenie Ruchu Focolari”, Paedagogia Christiana 2/36 (2015): 97-101.

${ }^{97}$ Lubich, La dottrina, 260.

${ }^{98}$ Marian Nowak, "Education as Support for the Integral Development of the Pupil", Paedagogia Christiana 2/42(2018): 11-32. 
della sua conoscenza ed esperienza, nonché una sorta di legame sociale ${ }^{99}$. Secondo Lubich la famiglia - comunità d'amore è un segno e un modello per ogni comunità umana. La ricchezza dei suoi valori di comunione, di solidarietà, di spirito di servizio e reciprocità può essere un punto di riferimento per l'ordine sociale. Perché ciò accada si deve plasmare nell'uomo la consapevolezza della sua natura sociale, la cui espressione fondamentale è il senso di comunità, cioè di comunione con altre persone. Educare, quindi, vuol dire condurre all'apertura e alla capacità di sacrificare se stesso per gli altri, fino a saperli amare come se stessi. Tale amore se diventa reciproco, secondo Lubich, genera l'unità ${ }^{100}$.

Una delle aree fondamentali per preparare un bambino alla vita sociale è il gioco attraverso il quale e nel quale egli esprime la sua attività. Lubich offre ai bambini il senso socio-etico del giocare proponendo i seguenti principi del cosiddetto Decalogo del divertimento e dei giochi: (1) Anche Gesù Bambino giocò. (2) Si giochi per fare la Volontà di Dio. (3) Anche nel gioco ci sia Gesù in mezzo. (4) Si giochi per far contento il fratello. (5) Si goda dei punti fatti dal fratello come dei propri. (6) Non si imiti il giocare del mondo perché spesso nel mondo il gioco è una piccola guerra. (7) Non ci si preoccupi del punteggio perché, data l'imperfezione umana, è umano errare. (8) Chi ci vede giocare veda in ogni mossa, in ogni sguardo, la Carità. (9) Si ricordi che il gioco non ha fine a se stesso, ma deve essere un piccolo anticipo del Paradiso. (10) Che dal Cielo Gesù e Maria vedano in noi, giocatori, i loro 'bambini' atti ad entrare del Regno dei Cieli ${ }^{101}$.

Particolarmente importante nella pedagogia di Lubich è un metodo che insegna ad amare gli altri, applicato universalmente nel Movimento: la cosiddetta 'arte d'amare'. Ella spiega che il termine "arte" indica che si tratta di acquisire determinate abilità. Indicando quest' "arte di amare" nel processo di educazione, autoeducazione e autoformazione, Lubich ne spiega tre caratteristiche principali che la definiscono. L'universalità, perché si richiede di amare tutti; la concretezza, perché bisogna amare attraverso le opere e non solo emotivamente; la reciprocità, perché è necessario amare in maniera tale da suscitare la capacità d'amare nell'altro. 'L'arte di amare' consiste nell'assumere uno specifico atteggiamento d'amore che allo stesso tempo viene

99 Wojciech Kajtoch, "Pisząc miłość", Polonistyka (2012): 102-103.

${ }^{100}$ Lubich, La dottrina, 267-269, 274-275, 320; anche: Chiara Lubich, La dottrina spirituale, ed. Michel Vandeleene (Milano: Mondadori, 2006), 320 [nota dell'autore: solamente per questa pubblicazione mi riferisco all'edizione della Mondadori].

${ }^{101}$ Lubich, Raccolta, 8. 
modellato e sviluppato. Lubich lo definisce in quattro passi, dando una spiegazione non solo di carattere religioso ma anche di natura universale.

(1) Amare tutti: così fa Dio che è Padre di tutti e quindi tutti gli uomini sono fratelli tra loro. Bisogna cominciare ad amare il prossimo in ogni attimo presente. Amarlo significa essere per lui come una madre: servire, donare il proprio tempo, saper sacrificarsi.

(2) Amare per primi: significa prendere l'iniziativa consapevoli di poter essere un dono per l'altro, nonostante la mancanza della sua risposta. Avere sempre lo sguardo puro, disinteressato.

(3) Amare come se stessi: ovvero volere per l'altro ciò che si vorrebbe per sé e non fare agli altri cio che non risulta piacevole. Lubich indica la cosiddetta 'Regola d'oro', presente nei sacri libri delle varie religioni. Nel cristianesimo essa dice: "Tutto quanto volete che gli uomini facciano a voi, anche voi fatelo a loro" (cf. Mt 7,12; Lc 6,31).

(4) Farsi uno: è una forma di profonda empatia che sta nel fare dentro di sé un certo vuoto per poter accogliere con amore l'altro con tutta la portata delle sue difficoltà, gioie, convinzioni e cultura.

Lubich ritiene che tale 'arte di amare' sia universale perché può essere vissuta da tutti: bambini e genitori, insegnanti e studenti, superiori e dipendenti, ma il punto di partenza deve sempre essere un impegno a rinnovare costantemente l'amore per cui la famiglia, la scuola e qualsiasi altro gruppo sociale possono diventare una vera comunità educativa ${ }^{102}$. Questa premessa trova consonanza con la visione del sociologo polacco Wojciech Świątkiewicz che afferma che Chiara Lubich, ponendo l'amore come il fondamento della vita familiare, contraddice la tesi secondo la quale il Vangelo indica un Regno di Dio inteso esclusivamente in senso religioso e, con E. Mounier, proclama: 'amo ergo sum'. Egli sottolinea che Lubich, dicendo che "nella famiglia è spontaneo vivere l'uno per l'altro, vivere l'altro", indica che la vocazione della famiglia è diventare: "il seme della società". Infatti tutto ciò è "il seme dell'accoglienza tra gruppi, popoli, tradizioni, razze e civiltà che apre alla reciproca inculturazione" 103 .

102 Cf. Chiara Lubich, Arte di amare (Roma: Città Nuova, 2005); Mariola T. Kozubek, Stanisław Grochmal, "Rodzina wspólnotą miłości w świetle pedagogii jedności Ruchu Focolari”, w: Wokót rodziny. Wychowanie, kultura, społeczeństwo, red. Andrzej Garbarz, Grzegorz Grzybek (Rzeszów: Wyd. i Drukarnia Diecezji Rzeszowskiej, 2011), 264-268.

103 Wojciech Świątkiewicz, “Amo, ergo sum”, Nowe Miasto 2-3 (2008): 169; cf. Lubich, La dottrina, 257, 269; Raul Augusto de Sousa Leite Silva, "A Utopia da Fraternidade Universal em Chiara Lubich", in: Ética relacional: um caminho de sabedoria, ed. Silvestre Ourives Marques, Miguel Panão, Pedro Vistas (Universidade Católica Editora, Lisboa 2017), 13-23. 


\subsection{L'amore come base dell'educazione a superare le difficoltà}

L'educazione in famiglia è il frutto dell'amore dei genitori che viene messo alla prova dalle difficoltà, dalle sofferenze e dalle sconfitte ad esso associate $^{104}$. Jorge Bergoglio, ancor da insegnante argentino, avvertiva i genitori di essere consapevoli dei rischi che comportava nell'educazione trascurare la dimensione del trascendente poiché in tale caso le cose diventano idoli e questi "si trasformano in demoni, distruggendo e divorando coloro che hanno intenzione di goderseli". Invitava genitori ed educatori a modellare "le idee chiare che potevano essere accettate dai giovani e dai bambini come proprie, per aiutarli a orientarsi sui percorsi della vita" 105 .

La dimora familiare, dove l'autorevolezza dei genitori si basa sull'amore che rafforza i legami, è la prima scuola d'amore per il bambino. In essa il bambino impara a saper vivere bene anche le difficoltà della vita ${ }^{106}$. E tale è l'esperienza di Chiara Lubich che, unita alla sua profonda fiducia in Dio, le ha permesso di sopravvivere anche alle atrocità della guerra, rendendola capace di accettare e superare difficoltà e sofferenze e, di conseguenza, educare le giovani generazioni ad affrontare le difficoltà della vita ${ }^{107}$.

I giovani del periodo a cavallo tra il XX e il XXI secolo sono, particolarmente, esposti all'influenza delle promesse edonistiche del raggiungimento della felicità senza sforzi. Lubich, invece, si situa nella dimensione della verità e della realtà, mostrando loro il significato più profondo di ogni sofferenza che sta nella sofferenza di Cristo crocifisso e spiega loro che:

Non si può amare il dolore di per sé, perché è un non essere: la malattia è una non salute; il dolore, l'angoscia, sono non gioie. Il dolore è sempre un negativo. Va amato, invece, Gesù crocifisso e abbandonato, il quale è presente in ogni sofferenza ed in ogni persona sofferente [...]. Si va in fondo al cuore e si dice: "Gesù, io voglio seguirti, anche in croce, anche abbandonato, e adesso ne ho

104 Giovanni Paolo II, Gratissimam, n. 16.

105 Bergoglio, Wymagania, 23.

${ }^{106}$ Por. Kornas-Biela, Rodzina; Katarzyna Olbrycht, "Wychowanie w rodzinie, jako wychowanie do wartości domu”, in: Dom, w którym rodzi się wspólnota. Rodzina, społeczeństwo, Kościót, ed. Mariola T. Kozubek (Katowice: Księgarnia św. Jacka, 2012), 55-69.

107 Lubich, Dottorati, 327. 
l'occasione" [...]. E poi ci si mette ad amare il fratello, o si continua a fare qualsiasi altra volontà di Dio ${ }^{108}$.

Il principio evangelico della spiritualità del Movimento che propone un significato per la sofferenza, si riflette nelle parole di Gesù espresse nel suo grido sulla croce: "Dio mio, Dio mio, perché mi hai abbandonato?" (Mt 27,46; $M c$ 15,3). Lubich sottolinea qui l'umanità di Gesù in cui Lui si identifica con tutta la sofferenza umana. Ella spiega che Gesù ha vinto il dolore dell'abbandono («Padre, nelle tue mani consegno il mio spirito», $L c$ 23,46), e quindi "ci insegna pure a vedere la difficoltà, l'ostacolo, la prova, l'impegno, l'errore, il fallimento, il dolore, come qualcosa da affrontare, da amare, da superare" 109 .

Alla base dell'educare alle difficoltà vi è l'amore impegnato, attuato ogni giorno e basato sul sacrificio, nella convinzione che "non si fa nulla di buono, di utile, di fecondo al mondo senza conoscere, senza sapere accettare la fatica, la sofferenza, [...] la croce" - dice Lubich ${ }^{110}$. Il fatto che la Chiesa cattolica abbia avviato i processi di beatificazione di diversi giovani del Movimento ${ }^{111}$, riconoscendo nei loro atteggiamenti la straordinaria capacità di amare il prossimo nella vita quotidiana, mostra che l'educazione al saper superare le difficoltà con l'amore porta alla maturità personale. Un esempio di tale realtà potrebbe essere la famiglia italiana di Maria Teresa e Ruggero Badano, la cui figlia unica Chiara Luce (1971-1990) nell'anno 2010 è stata proclamata beata in riconoscimento delle sue virtù eroiche ${ }^{112}$.

I coniugi Badano sono venuti in contatto con il Movimento dei Focolari nel 1981 quando la loro figlia Chiara aveva dieci anni. Come famiglia hanno partecipato al già menzionato Congresso di Famiglie Nuove - il Familyfest durante il quale Chiara Lubich ha tenuto un discorso sull'amore in famiglia ${ }^{113}$. Questo evento è stato per loro una tappa importante che ha contribuito all'approfondimento della loro vita coniugale. "Se qualcuno ci chiedesse quando

108 Chiara Lubich, Perché mi hai abbandonato. Il dolore nella spiritualità dell'unità, ed. Anna Fratta (Roma: Città Nuova, 1997), 4.

109 Lubich, Dottorati, 327.

110 Lubich, La dottrina, 50.

111 Tra gli altri: Maria Orsola Bussone (1954-1970), Alberto Michelotti (1958-1980), Carlo Grisolia (1960-1980), Daniela Zanetta (1962-1986), Santa Scorese (1968-1990).

${ }^{112}$ Livio Maritano, "Per decreto del Papa, Chiara Badano è venerabile", http://www. diocesiacqui.piemonte.it/badano.htm [20.01.2019]; Franz Coriasco, Dai tetti in giù. Chiara Luce Badano raccontata dal basso (Roma: Città Nuova, 2011), 113; Angelo Amato, "Omelia durante il Rito di Beatificazione", L'Osservatore Romano, 26 settembre 2010: 8.

${ }^{113}$ Cf. Lubich, La dottrina, 263-267. 
ci siamo sposati, risponderemmo: quando abbiamo conosciuto questo stile di vita"114. Per la figlia sono diventati ancor più "un modello di rispetto reciproco, calore e unità"'115. L'attuazione fedele della spiritualità dell'unità del Movimento dei Focolari ha reso la loro famiglia una vera comunità d'amo$\mathrm{re}^{116}$. E la vita evangelica e la formazione comunitaria ricevuta nel Movimento hanno gettato luce sulla loro genitorialità. Maria Teresa sottolinea che amavano incommensurabilmente la loro figlia, ma allo stesso tempo erano esigenti con lei, convinti che fosse anche figlia di Dio e come tale avrebbero dovuto educarla nella libertà ${ }^{117}$. Chiara è, così, cresciuta spiritualmente, intellettualmente e socialmente anche attraverso una formazione appropriata nella comunità degli adolescenti del Movimento. In essa, la giovane Badano, circondata da amore affettuoso, cura e sostegno, ha vissuto con dignità la sua esperienza della malattia dovuta ad un cancro ${ }^{118}$ che è stata una sfida per l'intera famiglia. Maria Teresa racconta che la malattia della figlia è stata una grande sofferenza per loro, genitori, ma la forza per superarla l'hanno presa dalla fiducia in Dio e dai consigli molto preziosi di Chiara Lubich ${ }^{119}$. La Fondatrice del Movimento ha accompagnato la giovane Chiara sin dall'inizio della malattia e lo scambio di corrispondenza lo dimostra in modo eloquente. Ne citiamo alcuni brani perché mostrano la maturità di questa relazione educativa che è un arricchirsi reciproco della propria umanità elevata a livello soprannaturale. Scrive Lubich: "Ti sono vicino, come ad una carissima Gen ${ }^{120}$.

114 Mariagrazia Magrini, Ze światła ku świattu. Błogosławiona Chiara Badano, trad. Anna Popławska (Kraków: Wyd. Św. Stanisława BM, 2011), 48.

115 Michele Zanzucchi, Mam wszystko. 18 lat życia Chiary Luce, trad. Zespół (Kraków: Fundacja Mariapoli, Wyd. M, 2004), 30 [Edizione italiana: «Io ho tutto». I diciotto anni di Chiara Luce (Roma: Città Nuova, 2010)].

116 Ibidem, 55. Ci sono nella spiritualità del Movimento cosiddetti "strumenti della spiritualità" tra cui quello della "comunione d'anima". Esso sta nello scambio, con chi si ha un certo legame, delle riflessioni di carrattere spirituale, ovvero che riguardano la meditazione fatta sulla parola del Vangelo e sugli effetti che esso porta nella propria vita [cf. Mariola T. Kozubek, "Wychowanie do wiary w rodzinie - propozycja duchowości jedności", Homo Dei 1 (2014): 23-36; Kozubek, Grochmal, "Rodzina”, 268-269].

117 Redakcja, "Błogosławiona Chiara Luce Badano", Nowe Miasto 5 (2010): 1. Inserto.

118 Mariola T. Kozubek, "Środowisko wychowawcze rodziny i Ruchu Focolari w doświadczeniu choroby bł. Chiary Luce Badano (1971-1990)", in: Cierpienie - tajemnica i wyzwanie, ed. Antoni Bartoszek (Katowice: Emmanuel, 2014), 142-160.

119 Joanna Bątkiewicz-Brożek, "Światło dla wszystkich”, Gość Niedzielny 9 (2014): 16.

${ }^{120}$ Nota d'Autore: Gen, abbreviazione di Generazione nuova, è il nome che disegna i giovani e le giovani appartenenti al Movimento dei Focolari. 
E ti assicuro della mia unità e della mia preghiera. Grazie per il tuo inestimabile sacrificio. Che Maria ti accompagni e ti benedica"121.

Prima della sua morte, la diciasettenne Chiara Luce svela la profonda dimensione interiore del dolore che sta vivendo. Scrive a Lubich:

Ho interrotto la chemioterapia perché non porta gli effetti: nessun risultato, nessun miglioramento. Così la medicina ha deposte le armi! Solo Dio può. Avendo interrotto la terapia provo l'aumento dei dolori della schiena, dovuti ai due interventi e dall'immobilità nel letto. Quasi non riesco a girarmi [...]. Riuscirò ad essere fedele a Gesù Abbandonato? [...] Mi sento molto piccola e la strada da percorrere è cosi difficile. Spesso mi sento soffocata dal dolore. Ma è lo Sposo che viene a trovarmi, vero? Sì, anch'io ripeto assieme a te: "Lo vuoi tu, Gesù, lo voglio anch'io"'122.

E la Fondatrice del Movimento le risponde:

Ti ringrazio per la tua foto. Il tuo volto irradiante esprime l'amore a Gesù. Chiara, non abbi paura di dirGli il tuo "si”" attimo dopo attimo. Lui ti darà la forza, sii sicura! Anch'io prego per questo e sono con te sempre. Dio ti ama immensamente e vuole penetrare nell'intimo della tua anima e farti sperimentare gocce di Cielo. Chiara Luce è il nome che ho pensato per te; ti piace? È la luce dell'Ideale che vince il mondo. Te lo mando con tutto il mio amore ${ }^{123}$.

In questo atteggiamento si può vedere una trascendenza verticale - come direbbe Wojtyła - perché "dalla forza di riferimento alla verità, dalla forza della coscienza" un giovane è in grado di controllare il proprio agire, le scelte e i desideri, ponendosi in certo senso "sopra di essi"124.

Nell'insegnamento del Concilio Vaticano II sull'educazione in famiglia si sottolinea che la vera educazione deve promuovere la formazione della persona umana anche in vista del suo fine ultimo ${ }^{125}$. Livio Maritano, vescovo di Acqui, durante il funerale di Chiara Badano ha sottolineato che lei era

121 Magrini, Ze światta, 144.

122 Zanzucchi, Mam wszystko, 64-65.

123 Ibidem.

124 Karol Wojtyła, Osoba i czyn oraz inne studia antropologiczne (Lublin: TN KUL, 2000), 486.

${ }^{125}$ Cf. Concilio Vaticano II, Dichiarazione sull'educazione cristiana Gravissimum educationis (Città del Vaticano: Libreria Editrice Vaticana, 1968), n. 1. 
figlia di una famiglia cristiana, di una comunità di credenti e del Movimento, dove si tende all'amore reciproco e ciò le ha permesso di collaborare con la grazia $^{126}$.

Lubich esprime la convinzione che per donare all'umanità la sua più alta dignità, che sta nel sentirsi un solo popolo, una famiglia e non un insieme di popoli, uno accanto all'altro, è indispensabile saper accettare la sofferenza per amore, la sofferenza che richiede l'amore ${ }^{127}$.

\section{Conclusioni}

Dalle analisi svolte emerge che per Chiara Lubich l'amore è un valore universale e perciò la persona umana si realizza nell'amore nonostante il proprio credo. Ella, mettendo l'amore alla base dell'educazione in famiglia, si apre alla dimensione trascendente, sia dell'educando che dell'educatore, e con tale impostazione il processo educativo si inserisce nel pensiero sull'educazione della corrente cristiana ove esso viene visto come la possibilità di condurre le persone a fare un dono reciproco della propria umanità. Il bambino, amato e amante, matura in se stesso un'idea dell'amore che influisce non solo sul suo sviluppo socio-emotivo, ma anche su quello cognitivo e con ciò il bambino diventa non solo un attore, ma in certo senso anche un creatore di una nuova cultura, in cui l'amore è una categoria culturale e cognitiva.

La specificità della pedagogia di Lubich sta nel condurre l'educando a sperimentare l'amore nelle sue varie manifestazioni - compreso il riconoscerlo nelle difficoltà e nella sofferenza - e nel saper realizzare creativamente questo amore nella vita quotidiana, attraverso le dinamiche della reciprocità modellate sulla relazione trinitaria. Secondo Lubich, l'educazione avviene in una tensione positiva tra: la famiglia, in quanto ambiente d'educazione di base; la comunità del Movimento compresa in senso lato e percepita come una grande famiglia; e il mondo esterno descritto da lei in quanto famiglia umana.

In tempi segnati dall'intensa migrazione di persone e dal pluralismo culturale, la visione di Lubich apporta un significativo valore aggiunto al processo educativo: considerando l'amore come dinamica fondamentale di questo processo ella prende in considerazione la formazione del bambino all'apertura verso l'alterità e verso la ricchezza dell'Altro, mentre allo stesso tempo sottolinea la necessità di approfondire l'identità individuale, familia-

\footnotetext{
126 Magrini, Ze światta, 206-207.

127 Lubich, La dottrina, 50.
} 
re, comunitaria, religiosa e culturale. Sorge però una domanda: se la fonte e il fondamento dottrinale del Movimento dei Focolari è il carisma riconosciuto dalla Chiesa cattolica, ma accolto in vari modi anche dai seguaci di altre religioni e da persone di convinzioni non religiose, - in tale pluralismo, sarà garantita l'adeguata preparazione delle persone che in quanto educatori e animatori sono coinvolti nella rete di formazione del Movimento stesso? Indubbiamente, per la teoria pedagogica sarebbe utile una ricerca empirica approfondita in questo ambito per poter discernere i vari aspetti del realismo pedagogico il quale è talvolta minacciato dall'approccio strettamente ideologico di tipo confessionale specie lì dove si tratta di una formazione basata sulla spiritualità.

Senza dubbio, l'esperienza pedagogica di Chiara Lubich e il pensiero pedagogico sull'amore nella famiglia che da essa deriva sono coerenti e mostrano il significato essenziale dell'esperienza di tipo religioso che rende sistematica la vita umana nelle sue varie dimensioni e specialmente in quella spirituale. In questo senso l'argomento presentato in questo articolo potrebbe essere d'ispirazione per il pensiero pedagogico polacco dato che nelle varie descrizioni esistenti sull'amore nell'educazione manca il riferimento significativo alla dimensione spirituale dell'uomo.

\section{Bibliografia}

Abignente, Lucia. Memoria e presente. La spiritualità del Movimento dei Focolari in prospettiva storica. Roma: Città Nuova, 2010.

Abignente, Lucia. Qui c'è il dito di Dio. Carlo de Ferrari e Chiara Lubich: il discernimento di un carisma. Roma: Città Nuova, 2017.

Amato, Angelo. "Omelia durante il Rito di Beatificazione". L'Osservatore Romano, 26 settembre 2010: 8.

Bagrowicz, Jerzy. Stawać się bardziej człowiekiem. U podstaw edukacji religijnej [Diventare più uomo. Alle basi dell'educazione religiosa]. Toruń: Wyd. Naukowe UMK, 2008.

Balthasar, von Hans Urs. La mia opera ed epilogo. Milano: Jaca Book, 1994.

Bartel, Sabina. "Autorytet rodzicielski" ["Autorevolezza genitoriale"]. In: Stownik matżeństwa i rodziny [Vocabolario sul matrimonio e sulla famiglia], ed. Edward Ozorowski, 31-32. Warszawa-Lomianki: ATK, 1999.

Bątkiewicz-Brożek Joanna. "Światło dla wszystkich" [“Luce per tutti”]. Gość Niedzielny 9 (2014): 16. 
Benedetto XVI. Enciclica Deus caritas est. Città del Vaticano: Libreria Editrice Vaticana, 2005.

Benedetto XVI. Lettera alla Diocesi e alla Città di Roma sul compito urgente dell'educazione. 21 gennaio 2008. Città del Vaticano: Libreria Editrice Vaticana, 2008.

Benedetto XVI. Messaggio ai partecipanti al II Congresso Mondiale dei Movimenti ecclesiali e delle Nuove comunità. 22 Maggio 2006. Città del Vaticano: Libreria Editrice Vaticana, 2006.

Bergoglio, Jorge Mario. Wymagania i pasja. O wychowaniu chrześcijańskim [Esigenze e passione. Sull'educazione cristiana]. Trad. Lidia Wrona. Kraków: Espirit $\mathrm{SC}, 2013$.

Bianco, Gianni. Una famiglia grande come il mondo. Roma: Città Nuova, 2008.

"Błogosławiona Chiara Luce Badano" ["Beata Chiara Luce Badano"]. Nowe Miasto 5 (2010): 1. Inserto.

Carella, Nino. Silvia prima di Chiara. La ricercha di una strada nuova. Roma: Città Nuova, 2014.

Centro Chiara Lubich, Istituto Universitario Sophia. Dottorati honoris causa conferiti a Chiara Lubich. Laudationes, Motivazioni, Lezioni magistrali. Ed. Florence Gillet e Rosalia Parlapiano. Roma: Città Nuova, 2016.

Centro San Giovanni Bosco. Storia istituzionale. Rocca di Papa: Archivio Chiara Lubich.

Coda, Piero. "L'idea di Università di Chiara". In: La fonte e le frontiere. Sophia compie 10 anni, 125-165. Roma: Città Nuova, 2018.

Coriasco, Franz. Dai tetti in giù. Chiara Luce Badano raccontata dal basso. Roma: Città Nuova, 2011.

Concilio Vaticano II. Costituzione pastorale sulla chiesa nel mondo contemporaneo Gaudium et spes. Città del Vaticano: Libreria Editrice Vaticana, 1968.

De Beni, Michele. Comunicare per amare. Roma: Città Nuova, 2005.

De Beni, Michele. Essere educatori. Corraggio di una presenza. Roma: Città Nuova, 2013.

Folonari, Giuglia Eli. Lo spartito scritto in cielo. Cinquant'anni con Chiara Lubich. Roma: Città Nuova, 2012.

Foresi, Pasquale. "Educatori a convegno". Città Nuova 1 (1960): 6-7.

Francesco. Ai partecipanti alla plenaria della Congregazione per L'Educazione Cattolica. Sala Clementina, 13 Febbraio 2014. Città del Vaticano: Libreria Editrice Vaticana, 2014.

Francesco. Discorso ai Partecipanti all'Asemblea Generale del Movimento dei Focolari. Sala Clementina, Venerdi, 26 settembre 2014. Città del Vaticano: Libreria Editrice Vaticana, 2014. 
Francesco. Esortazione apostolica postsinodale Amoris Laetitia. Città del Vaticano: Libreria Editrice Vaticana, 2015.

Francesco. Festa delle Famiglie. Discorso del santo Padre Francesco. Croke Park Stadium (Dublino). Sabato, 25 agosto 2018. Città del Vaticano: Libreria Editrice Vaticana, 2018.

Francesco. Incontro con la comunità del Movimento dei Focolari. Sagrato del Santuario Maria Theotokos a Loppiano (Firenze). Giovedi, 10 maggio 2018. Città del Vaticano: Libreria Editrice Vaticana, 2018.

Francesco. La Famiglia. Educazione. Udienza generale, 20.05.2015. Città del Vaticano: Libreria Editrice Vaticana, 2015.

Fromm, Erich. Avere o essere. Milano: Mondadori, 1977.

Fromm, Erich. L'arte di amare. Milano: Mondadori, 1986.

Furmanek, Waldemar. Miłość - zagubiona wartość współczesnej pedagogiki [L'Amore - un valore perduto nella pedagogia moderna]. Rzeszów: FOSZE, 2011.

Furmanek, Waldemar. Rozwój rozumienia miłości [Lo sviluppo della comprensione dell'amore]. Rzeszów: Wyd. Oświatowe FOSZE, 2011.

Galarowicz, Jan. Na ścieżkach prawdy. Wprowadzenie do filozofii [Sui sentieri di verità. Introduzione alla filosofia]. Kraków: PAT, 1992.

Giordani, Igino. Famiglia comunità d'amore. Roma: Città Nuova, 1969.

Giordani, Igino. La famiglia. Ricordi, pensieri. Roma: Città Nuova, 2005.

Giordani, Igino. Memorie di un cristiano ingenuo. Roma: Città Nuova, 1981.

Giovanni Paolo II. Lettera alle famiglie. Gratissimam sane. Città del Vaticano: Libreria Editrice Vaticana, 1994.

Giovanni Paolo II. Discorso ai Membri del Movimento dei Focolari. Centro internazionale «Mariapoli»-Rocca di Papa, Domenica, 19 agosto 1984. Città del Vaticano: Libreria Editrice Vaticana, 1984.

Giovanni Paolo II. Discorso all'Organizzazione delle Nazioni Unite per L'Educazione, la Scienza e la Cultura (UNESCO). Parigi, 2 giugno 1980. Città del Vaticano: Libreria Editrice Vaticana, 1980.

Giovanni Paolo II. Esortazione apostolica 'Familiaris consortio'. Città del Vaticano: Libreria Editrice Vaticana, 1981.

Guglielmoni, Luigi, Fausto Negri. L'amore familiare secondo san Paolo. Leumann: ELLEDICI, 2009.

Horowski, Jarosław. "Rola rodziny w wychowaniu seksualnym dzieci i młodzieży (w Świetle dokumentów Stolicy Apostolskiej)" ["Il ruolo della famiglia nell'educazione sessuale dei bambini e giovani (alla luce dei documenti della Santa Sede)"]. Paedagogia Christiana 1/9 (2002): 179-188. 
Jankowski, Wiesław. "Znaczenie wychowania integralnego dla odnowy rodziny" [Il significato dell'educazione integrale per un rinnovamento della famiglia]. Studia nad Rodzina ATK 1 (1997): 73-84.

Katolickie poradnictwo rodzinne w Polsce w r. 2016 [La consulenza famigliare cattolica per le famiglie in Polonia nell'anno 2016]. Warszawa: Instytut Statystyki Kościoła Katolickiego, 2017.

Kiereś, Barbara. "Rodzina a problem aktualizacji przyrodzonej religijności człowieka. Na kanwie A.M. Krąpca realistycznej filozofii człowieka" ["La famiglia e il problema dell'attualizzazione della religiosità primaria dell'uomo. Alla luce della filosofia realistica dell'uomo di A.M. Krapiec"]. In: Edukacyjny potencjat religii [Il potenziale educativo della religione], ed. Jerzy Bagrowicz, Jarosław Horowski, 241-257. Toruń: Wydawnictwo Naukowe UMK, 2012.

Kim, Colomba In Hye. Gli sposi e la famiglia in Igino Giordani. Pensiero e testimonianza. Roma: Città Nuova, 2011.

Kłos-Skrzypczak, Aleksandra. "Nauki o rodzinie w perspektywie amerykańskiego Szkolnictwa wyższego" ["Family study alla luce degli studi universitari negli Stati Uniti”]. Studia Teologiczne i Humanistyczne 1/2 (2011): 247-258.

Kornas-Biela, Dorota. Rodzina: źródto życia i szkoła mitości [La famiglia: fonte della vita e scuola dell'amore]. Lublin: TN KUL, 2000.

Kozubek, Mariola T. "Pedagogie współczesnych ruchów odpowiedzią na Familiaris consortio" ["Le pedagogie dei movimenti moderni in risposta alla Familiaris consortio"]. Studia Teologiczne i Humanistyczne 1/2 (2011): 195-209.

Kozubek Mariola T. "Środowisko wychowawcze rodziny i Ruchu Focolari w doświadczeniu choroby bł. Chiary Luce Badano (1971-1990)" ["L'ambiente educativo della famiglia e del Movimento dei Focolari nell'esperienza di malattia della beata Chiara Luce Badano (1971-1990)"]. In: Cierpienie - tajemnica $i$ wyzwanie [La sofferenza - il mistero e la sfida], ed. Antoni Bartoszek, 142-160. Katowice: Emmanuel, 2014.

Kozubek, Mariola T. "Wspólnota i jedność w wybranych obszarach życia społecznego - doświadczenie Ruchu Focolari”. [“Comunità e unità in alcune aree della vita sociale - l'esperienza del Movimento dei Focolari']. Paedagogia Christiana 2/36 (2015): 97-101. DOI: http://dx.doi.org/10.12775/PCh.2015.027.

Kozubek, Mariola T. "Wychowanie do wiary w rodzinie - propozycja duchowości jedności" ["Educazione in famiglia alla fede - una proposta della pedagogia dell'unità"]. Homo Dei 1(2014): 23-36.

Kozubek, Mariola T., Stanisław Grochmal. "Rodzina wspólnotą miłości w świetle pedagogii jedności Ruchu Focolari" ["Famiglia - comunità d'amore alla luce della pedagogia dell'unità del Moviemnto dei Focolari']. In: Wokót rodziny. Wychowanie, kultura, społeczeństwo [Attorno alla famiglia. Educazione, cultu- 
ra, società], ed. Andrzej Garbarz, Grzegorz Grzybek, 255-273. Rzeszów: Wyd. i Drukarnia Diecezji Rzeszowskiej, 2011.

Krąpiec, Mieczysław A. Ja-czlowiek [Io-uomo]. Lublin: KUL, 1998.

Lubich, Chiara. Ai Gen 3.1971-1975. Roma: Città Nuova, 1979.

Lubich, Chiara. Ai Gen 3.1975-'80. Roma: Città Nuova, 1994.

Lubich, Chiara. Ai Gen 3.1981-1995. Roma: Città Nuova, 2006.

Lubich, Chiara. Arte di amare. Roma: Città Nuova, 20055.

Lubich, Chiara. "Chi beve l'acqua pensa alla sorgente". In: Da Trento al mondo, dal mondo a Trento. Chiara Lubich nella sua città, ed. Michele Zanzucchi, 77-83. Roma: Città Nuova, 2002.

Lubich, Chiara. Colloqui con i GEN. Anni 1966/69. Roma: Città Nuova, 1998.

Lubich, Chiara. Colloqui con i GEN. Anni 1970/74. Roma: Città Nuova, 1999.

Lubich, Chiara. Colloqui con i GEN. Anni 1975/2000. Roma: Città Nuova, 2001.

Lubich, Chiara. Dove la vita si accende. Dialoghi sulla famiglia. Roma: Città Nuova, 1999.

Lubich, Chiara. "Igino Giordani: il confondatore". Nuova Umanita 1 (1995): 5-10.

Lubich, Chiara. La dottrina spirituale. Nuova edizione aggiornata e amplita, ed. Michele Vandeleene. Roma: Città Nuova, 2009.

Lubich, Chiara. Lettere dei primi tempi (1943-1949) alle origini di una nuova spiritualità. Roma: Città Nuova, 2010.

Lubich, Chiara. "Meditazioni” (1959). In: Chiara Lubich, Scritti Spirituali/1. Roma: Città Nuova, 1991 '

Lubich, Chiara. Perché mi hai abbandonato. Il dolore nella spiritualità dell'unità, ed. Anna Fratta. Roma: Città Nuova, 1997.

Lubich, Chiara. Raccolta dei testi di Chiara ai ragazzi o sui ragazzi. Stralci da conversazioni, lettere, messaggi dal 1953 ad oggi per la formazione degli animatori del Movimento Ragazzi per l'unità, ed. Centro Mondiale Ragazzi per l'unità. Rocca di Papa, 1999.

Lubich, Chiara. Tylko jedno. Katowice: Księgarnia św. Jacka, 1984.

Lubich, Chiara. Una via nuova. La spiritualità dell'unità. Roma: Città Nuova, 2002. Magrini, Mariagrazia. Ze światta ku świattu. Błogosławiona Chiara Badano, trad. Anna Popławska. Kraków: Wyd. Św. Stanisława BM, 2011. Ed. Italiana: Magrini Mariagrazia. Di luce in luce. Torino: Edizioni San Paolo, 2008.

Maritano, Livio. Per decreto del Papa, Chiara Badano è venerabile. http://www. diocesiacqui.piemonte.it/badano.htm [20.01.2019].

Morán, Jesús. “Trasferirsi nel fratello': una radicalizzazione antropologica”. In: Guardare tutti i fiori. Da una pagina del '49 di Chiara Lubich, ed. Lucia Abignente, Maria Caterina Atzori ed. altri, 39-51. Roma: Città Nuova, 2014. 
Mounier, Emanuel. Wprowadzenie do egzystencjalizmów [Introduzione agli esistenzialismi]. Kraków: ZNAK, 1964.

Nowak, Marian. "Education as Support for the Integral Development of the Pupil". Paedagogia Christiana 2/42 (2018): 11-32. DOI: http://dx.doi.org/10.12775/ /PCh.2018.013.

Nowak, Marian. "Wymiary teorii i praktyki w pedagogice w ujęciu Karola Wojtyły" ["Le dimensioni di teoria e di praxi in pedagogia nell'approccio di Karol Wojtyła"]. Wychowanie na co dzień 4-5(2005): 11-12.

Olbrycht, Katarzyna. "Co może odnaleźć pedagog w zbiorze tekstów «Charyzmat jedności» Chiary Lubich?" ["Che cosa può trovare un pedagogo nel libro «La dottrina spirituale» di Chiara Lubich"]. Nowe Miasto 2-3 (2008): 168.

Olbrycht, Katarzyna. "Wychowanie w rodzinie, jako wychowanie do wartości domu" ["Educazione in famiglia, come educazione al valore della casa"]. In: Dom, w którym rodzi się wspólnota. Rodzina, społeczeństwo, Kościót [La casa dove nasce la comunità: famiglia, società, chiesa], ed. Mariola T. Kozubek, 55-69. Katowice: Księgarnia św. Jacka, 2012.

Opozda, Danuta. "Integral Upbringing as the Fundamental Category of Practice and Theory of Pedagogy of Family in Christian Inspiration". Paedagogia Christiana 2/42 (2018): 151-164.

Ożóg, Tomasz. "Wychowanie chrześcijańskie w kościelenych ruchach religijnych" ["Educazione cristiana nei movimenti ecclesiali"]. In: Wychowanie chrześcijańskie. Między tradycja a wspólczesnościa [Educazione cristiana. Tra la tradizione e la modernità], ed. Alina Rynio, 953-963. Lublin: Wyd. KUL, 2007.

Paolo VI al Movimento dei Focolari. Roma: Città Nuova, 1978.

Półtawska, Wanda. "Prawidłowy start" ["Una partenza corretta"]. In: Miłość, matżeństwo, rodzina [Amore, matrimonio, famiglia], ed. Franciszek Adamski, 21-71. Kraków: Wyd. Apostolstwa Modlitwy, 1978.

Półtawska Wanda. "Rola rodziców w wychowaniu seksualnym młodzieży" ["Il ruolo dei genitori nell'educazione sessuale dei ragazzi"]. In: Wychowanie w rodzinie [Educazione in famiglia], ed. Franciszek Adamski, 193-208. Kraków: Petrus 2010.

Półtawska Wanda. Uczcie się kochać [Imparate ad amare]. Poznań: Edycja Świętego Pawła, 2015.

Silva, Raul Augusto de Sousa Leite. "A Utopia da Fraternidade Universal em Chiara Lubich". In: Ética relacional: um caminho de sabedoria, ed. Silvestre Ourives Marques, Miguel Panão, Pedro Vistas, 13-23. Lisboa: Universidade Católica Editora, 2017.

Słowik, Małgorzata. "O potrzebie rozwoju poradnictwa rodzinnego opartego na wartościach chrześcijańskich - na przykładzie działaności Kościoła katolickie- 
go w Polsce" ["Sul bisogno dello svillupo della consulenza famigliare, basata sui valori cristiani - l'esempio dell'attività della Chiesa cattolica in Polonia"]. Paedagogia Christiana 2/30 (2012): 193-213.

Stróżewski, Władysław. „Oblicza miłości” [“I volti dell’amore”]. Tygodnik Powszechny 6 (2000): 1.

Styczeń, Tadeusz. "Kochaj, gdyż Bóg nas wszystkich ukochał. Z Chiarą Lubich założycielką Ruchu Focolari rozmawia Tadeusz Styczeń" ["Ama, perchè Dio ci ha amati tutti. Con Chiara Lubich, la fondatrice del Movimento dei Focolari dialoga Tadeusz Styczeń"]. ETHOS 29 (1995): 17-22.

Sorgi, Tommaso. "Scuole del nostro tempo". Città Nuova 2 (1960): 11-12.

Świątkiewicz, Wojciech. “Amo, ergo sum”. Nowe Miasto 2-3 (2008): 169.

Świątkiewicz, Wojciech. "Między rodziną a życiem publicznym - ciągłość i zmiana orientacji na wartości w polskim społeczeństwie" ["Tra famiglia e vita pubblica - la continuità e il cambiamento della società polacca nell'orientamento verso i valori"]. In: Postawy spoleczno-religijne Polaków 1991-2012 [Atteggiamenti socio-religiosi dei polacchi 1991-2012], ed. Lucjan Adamczuk, Elżbieta Firlit, Witold Zdaniewicz, 192-194. Warszawa: Instytut Statystyki Kościoła Katolickiego SAC, 2013.

Tarnowski, Janusz. "Wprowadzenie do Deklaracji o wychowaniu chrześcijańskim". ["Introduzione alla Dichiarazione sull'educazione cristiana"]. In: Sobór Watykański II, Konstytucje, Dekrety, Deklaracje, 303-312. Poznań: Pallotinum, 1968.

Torno, Armando. PortarTi il mondo fra le braccia - Vita di Chiara Lubich. Roma: Città Nuova, 2011.

Tratti di storia del Movimento Famiglie Nuove, ed. Danilo e Anna Maria Zanzucchi. Roma: Città Nuova, 2005.

Voce, Maria. "Uno strumento efficace di formazione alla cultura dell'unità". In: $L a$ fonte le frontiere. Sophia compie 10 anni, 17-21. Roma: Città Nuova, 2018.

Wodarczyk Adam. Prorok żywego Kościoła [Il profeta della Chiesa viva]. Katowice: Emmanuel, 2008.

Wojtyła, Karol. "Amore e responsabilità". In: Karol Wojtyla, Metafisica della persona. Tutte le opere filosofiche e saggi integrativi, 478-495. Milano: Bompiani, 2003.

Wojtyła, Karol. Osoba i czyn oraz inne studia antropologiczne [Persona e atto e altri studi antropologici]. Lublin: TN KUL, 2000.

Wojtyła, Karol. "Teoria-prassi: un tema umano e cristiano". Rassegna di Pedagogia. Pädagogische Umschau 1-4 (2004): 17.

Zambonini, Franca. L'avventura dell'unità. Milano: Paoline Cinisello Balsamo, 1991. 
Zanhgi, Giuseppe M. "Pedagogia nuova". Città Nuova 2 (1960): 11.

Zanzucchi, Michele. Mam wszystko. 18 lat życia Chiary Luce, trad. Zespół. Kraków: Fundacja Mariapoli, Wyd. M, 2004. Ed. Italiana: «Io ho tutto». I diciotto anni di Chiara Luce. Roma: Città Nuova, 2010.

Żyliński, Jarosław. Mitość $i$ wychowanie [L'amore e l'educazione]. Poznań: W Drodze, 2016. 